\title{
Extensive optical and near-infrared observations of the nearby, narrow-lined type Ic SN 2007gr: days 5 to 415^
}

\author{
D. J. Hunter ${ }^{1}$, S. Valenti ${ }^{1}$, R. Kotak ${ }^{1}$, W. P. S. Meikle ${ }^{2}$, S. Taubenberger ${ }^{3}$, A. Pastorello ${ }^{1}$, S. Benetti ${ }^{4}$, V. Stanishev ${ }^{5}$, \\ S. J. Smartt ${ }^{1}$, C. Trundle ${ }^{1}$, A. A. Arkharov ${ }^{6,7}$, F. Bufano ${ }^{4}$, E. Cappellaro ${ }^{4}$, E. Di Carlo ${ }^{8}$, M. Dolci ${ }^{8}$, N. Elias-Rosa ${ }^{9}$, \\ S. Frandsen ${ }^{10}$, J. U. Fynbo ${ }^{11}$, U. Hopp ${ }^{12,13}$, V. M. Larionov ${ }^{6,7}$, P. Laursen ${ }^{11}$, P. Mazzali ${ }^{3,4,14}$, H. Navasardyan ${ }^{4}$, \\ C. Ries ${ }^{12}$, A. Riffeser ${ }^{12}$, L. Rizzi ${ }^{15}$, D. Y. Tsvetkov ${ }^{16}$, M. Turatto ${ }^{4}$, and S. Wilke ${ }^{12}$
}

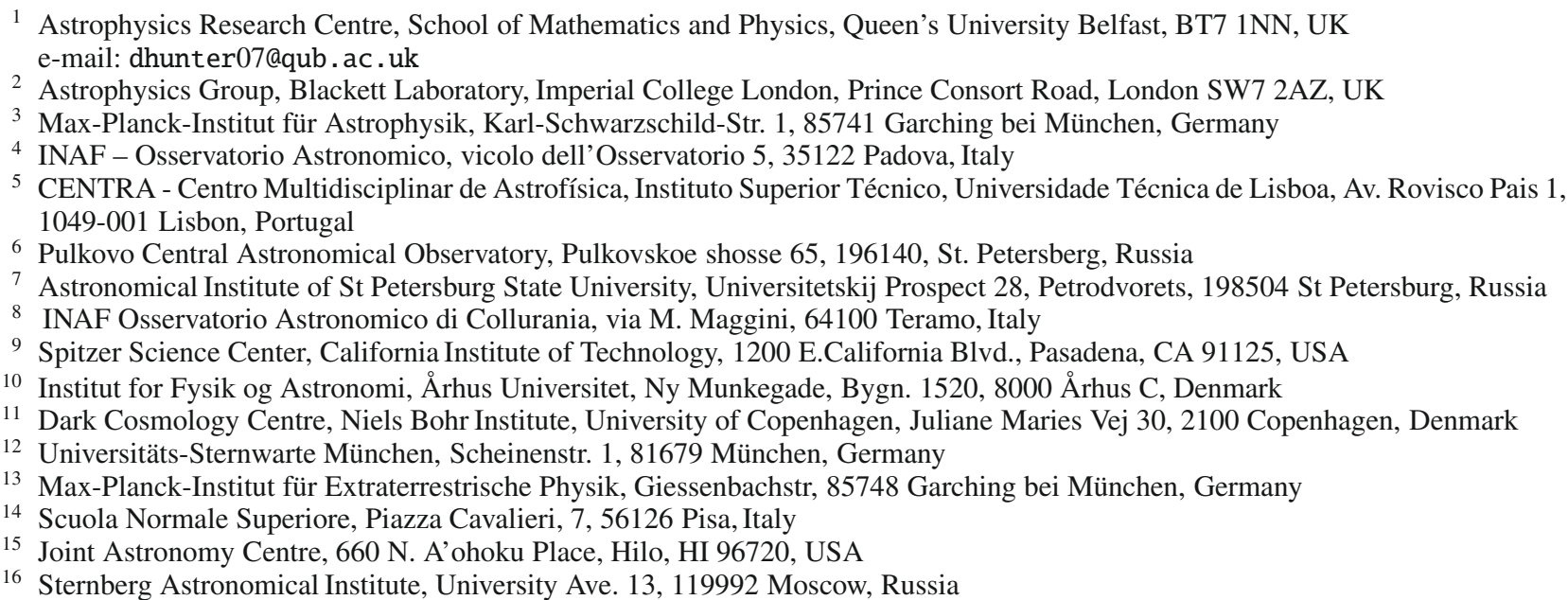

Received 15 July 2009 / Accepted 17 September 2009

\section{ABSTRACT}

\begin{abstract}
We present photometric and spectroscopic observations at optical and near-infrared wavelengths of the nearby type Ic supernova 2007gr. These represent the most extensive data-set to date of any supernova of this sub-type, with frequent coverage from shortly after discovery to more than one year post-explosion. We deduce a rise time to $B$-band maximum of $11.5 \pm 2.7 \mathrm{~d}$. We find a peak $B$-band magnitude of $M_{B}=-16.8$, and light curves which are remarkably similar to the so-called "hypernova" SN 2002ap. In contrast, the spectra of SNe 2007gr and 2002ap show marked differences, not least in their respective expansion velocities. We attribute these differences primarily to the density profiles of their progenitor stars at the time of explosion i.e. a more compact star for SN 2007gr compared to SN 2002ap. From the quasi-bolometric light curve of SN $2007 \mathrm{gr}$, we estimate that $0.076 \pm 0.010 M_{\odot}$ of ${ }^{56} \mathrm{Ni}$ was produced in the explosion. Our near-infrared (IR) spectra clearly show the onset and disappearance of the first overtone of carbon monoxide $(\mathrm{CO})$ between $\sim 70$ to $175 \mathrm{~d}$ relative to $B$-band maximum. The detection of the $\mathrm{CO}$ molecule implies that ionised He was not microscopically mixed within the carbon/oxygen layers. From the optical spectra, near-IR light curves, and colour evolution, we find no evidence for dust condensation in the ejecta out to about $+400 \mathrm{~d}$. Given the combination of unprecedented temporal coverage, and high signal-to-noise data, we suggest that SN $2007 \mathrm{gr}$ could be used as a template object for supernovae of this sub-class.
\end{abstract}

Key words. supernovae: individual: SN 2007gr - techniques: miscellaneous - supernovae: individual: SN 2002ap supernovae: general

\section{Introduction}

One of the main objectives of supernovae $(\mathrm{SNe})$ research is to understand the relation between the physics of the explosion and the nature of the progenitor star and its immediate environment.

Type I SNe, i.e. those lacking spectroscopic signatures of hydrogen, are divided into the Ia, Ib, and Ic categories. Of these, type Ia SNe constitute a physically distinct class and are

* Tables A.1 to A.5 are only available in electronic form at the CDS via anonymous ftp to cdsarc.u-strasbg.fr (130.79.128.5) or via

http://cdsweb.u-strasbg.fr/cgi-bin/qcat?J/A+A/508/371 believed to arise from the thermonuclear explosion of an accreting carbon-oxygen white dwarf. Instead, both the type Ib (He-rich) and the type Ic (He-poor) $\mathrm{SNe}$ are believed to originate from the core-collapse of stars more massive than $\sim 8 M_{\odot}$. $\mathrm{SNe}$ of type Ib and type Ic are quite heterogeneous in their spectral properties and energetics, which range from the relatively low kinetic energy $\left(\sim 10^{51} \mathrm{erg}\right)$ events like SN 1994I (Nomoto et al. 1994), to the high kinetic energy $\left(\sim 10^{52-53} \mathrm{erg}\right)$ broad-lined events sometimes dubbed "hypernovae" e.g. the broad-lined type Ic SNe 1998bw (Galama et al. 1998; Iwamoto et al. 1998) and 2003dh (Hjorth et al. 2003; Mazzali et al. 2003). These latter 
SNe have been associated with long-duration gamma-ray bursts (GRBs) (see Woosley \& Bloom 2006, for a recent review).

At present, there is no consensus regarding the differences between the progenitors that produce type Ib and Ic SNe. However, it is generally accepted that Wolf-Rayet stars are the most promising candidates as these have shed their outer layer of $\mathrm{H}$, as well as varying amounts of their He layer (Wheeler \& Levreault 1985). The $\mathrm{H}$ and He layers are primarily shed through strong stellar winds, resulting in WC and WO stars (i.e. carbon and oxygen dominated, respectively). The same massloss mechanism may likewise only remove the $\mathrm{H}$ layers so that the potential progenitors of type Ib $\mathrm{SNe}$ i.e. WN stars (nitrogen dominating over carbon) exhibit the products of core-H burning. Alternatively, the outermost layers may be stripped off by a companion star (Podsiadlowski et al. 1992) with the degree of envelope-stripping depending on the configuration of the binary system (Pols \& Nomoto 1997).

The distinction between type Ib and type Ic SNe is historical, and challenged by the idea that He may be present in both types. The detection of spectral features due to He in type Ib SNe might simply imply that the element is high in abundance and/or a sufficient quantity of radioactive nickel is mixed into the He layer, providing an excitation source (Wheeler et al. 1987; Shigeyama et al. 1990; Hachisu et al. 1991). The converse may be true for type Ic SNe explaining the non-detection of optical He I lines in type Ic spectra (Nomoto et al. 1990; Hachisu et al. 1991). Most important, no type Ic SN has yet displayed the isolated near-IR He line at $2.058 \mu \mathrm{m}$ to a strength comparable to those detected in type $\mathrm{Ib} \mathrm{SNe}$.

Consequently, it is possible that a continuous degree of $\mathrm{He}$ abundances and/or excitation exists from type Ib to Ic SNe, making the distribution smooth rather than bimodal. This seems consistent with the identification of intermediate cases such as SN 1999ex which was characterised by weak optical He I lines, and strong He I $\lambda \lambda$ 10830, 20581 lines in the near-IR (Hamuy et al. 2002). Additionally, the transitional nature of SNe such as SN 2005bf (Folatelli et al. 2006) and SN 2008D (Soderberg et al. 2008; Mazzali et al. 2008; Malesani et al. 2009), both of which underwent a metamorphosis from a type Ic at early times to a type Ib at later epochs, may also provide an insight into the controversy of He. The current challenge lies in linking the observed variations of type $\mathrm{Ib} / \mathrm{c} \mathrm{SNe}$ to the physical properties of their progenitor systems.

This paper presents the results of an intensive observational follow-up campaign of the type Ic SN $2007 \mathrm{gr}$ from shortly after explosion to more than a year later. The layout of this paper is as follows: in Sect. 2 we provide some basic information on SN 2007gr and its host galaxy, with the data acquisition and reduction procedures described in Sect. 3. This is followed in Sect. 4 by a description of the optical and near-IR photometric behaviour, including the colour evolution and quasi-bolometric light curve. Section 5 is devoted to the spectroscopic evolution; we end with a summary in Sect. 6.

\section{SN 2007gr}

SN 2007gr was discovered during the course of the Lick Observatory Supernova Search (LOSS) on 2007 August 15.51 UT (Madison \& Li. 2007). The SN exploded about 24 .' $^{\prime} \mathrm{W}$ and $155^{\prime \prime} 8 \mathrm{~N}$ of the nucleus of the nearby spiral galaxy NGC 1058. Chornock et al. (2007) classified SN 2007gr to be of type Ib/c from a spectrum taken soon after explosion. The classification was later refined to type Ic as the presence of He could not be confirmed in subsequent spectra (Valenti et al. 2008a).

Given that the object was undetected in an image taken on 2007 August 10.44 (unfiltered mag < 18.9; Madison \& Li. 2007), the explosion date can be confined to less than 5 days before discovery. Here, we adopt 2007 August 13 (JD $2454325.5 \pm 2.5$ ) as the explosion epoch, and 2007 August 25 (JD $2454337.0 \pm 1.0$ ) as the time of $B$ band maximum, used hereafter as the reference epoch, $t=0$ days (see Sect. 4.1). Although no source was detected at the location of the SN in HST pre-explosion images (Crockett et al. 2008), the $\mathrm{SN}$ was suggested to be a cluster member based on its close location to a bright source. The pre-explosion HST and ground based images marginally favour a young cluster with a turn-off mass of $28 \pm 4 M_{\odot}$.

The host galaxy of SN 2007gr, NGC 1058, belongs to a group of nearby galaxies of which NGC 925 is also a member. The distance to NGC 925 has been derived by Silbermann et al. (1996) as $9.29 \pm 0.69 \mathrm{Mpc}(\mu=29.84 \pm 0.16)$ using Cepheid variables; we adopt this value throughout the paper for the distance to SN $2007 \mathrm{gr}$. We note that an alternative distance estimate based on the Tully-Fisher relation has also been reported (10.6 $\pm 1.3 \mathrm{Mpc}$; Terry et al. 2002). The galaxy of SN $2007 \mathrm{gr}$ also hosted two prior supernovae, SN 1969L $\left(A_{V}=0.17\right.$; Burstein \& Heiles 1984) and SN 1961V $\left(A_{V} \sim 2.2-4.1\right.$; Filippenko et al. $1995 \mathrm{a}$ ) with the latter possibly being the outburst of a luminous blue variable (Goodrich et al. 1989).

We adopt a galactic extinction of $E(B-V)=0.062$ (Schlegel et al. 1998), and a host galaxy extinction of $E(B-V)=0.03$ which was estimated using the equivalent widths of $\mathrm{NaI} D$ absorption lines in a medium-resolution spectrum (Valenti et al. 2008a). We assume a total uncertainty of $20 \%$ for our estimate of the total extinction $E(B-V)_{\mathrm{tot}}=0.092 \pm 0.018 \mathrm{mag}$.

\section{Data acquisition and reduction}

The proximity of SN 2007gr lent itself to an extensive observational campaign starting 6 days before maximum light and extending through to +404 days. The data acquisition and reduction procedures are detailed below.

\subsection{Photometry}

UBVRI photometry of $\mathrm{SN} 2007 \mathrm{gr}$ was obtained from 2007 Aug. 18 to 2008 Sep. 30, spanning approximately -6 to +404 days with respect to $B$-band maximum.

The basic reduction of the data i.e., trimming, bias and overscan correction, and flat-fielding was performed using standard procedures within the IRAF environment. Images taken on the same night with the same filter were combined to improve the signal-to-noise ratio.

The instrumental magnitudes of SN $2007 \mathrm{gr}$ were obtained via the PSF fitting technique as implemented in the SNOOPY ${ }^{1}$ software package. This technique was preferred over aperture photometry because of the complex location of SN 2007gr, close to two bright sources (see inset Fig. 1).

Only for the observations taken on 2008 Feb. 10, did we subtract a template image of the host galaxy before performing our measurements on the SN. This was necessary given the poor

\footnotetext{
1 SNOOPY was originally devised by Patat (1996) and implemented in IRAF by Cappellaro. The package is based on DAOPHOT and has been optimized for SNe.
} 


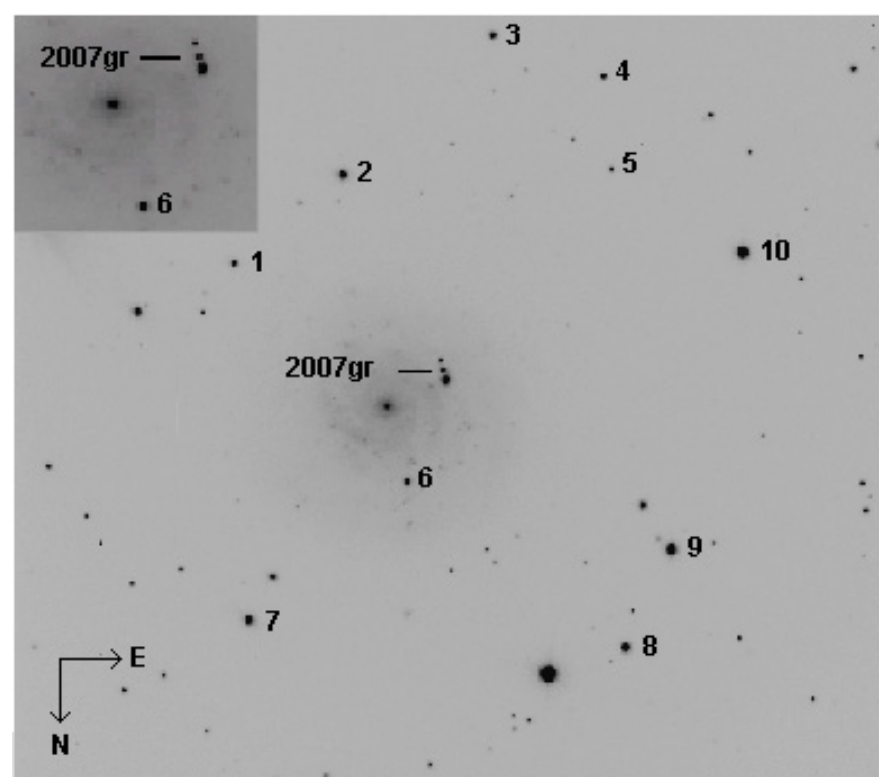

Fig. 1. Section of $V$-band image taken on 2008 Jan. $13(\sim+142.4 \mathrm{~d}$ relative to $B$ maximum) with the Nordic Optical Telescope (NOT). SN $2007 \mathrm{gr}$ and the local sequence of stars are marked. The field of view is $6 ! 3 \times 6 ! 3$. The inset shows the two bright stars that flank SN $2007 \mathrm{gr}$, both of which are approximately $5^{\prime \prime}$ from the SN.

seeing conditions ( $>1$ ". 8$)$. For the template, we used archival images obtained on 2005 Jan. 13 with the Isaac Newton Telescope.

The galaxy subtraction was performed using a purposewritten script based on the ISIS package (Alard 2000; Alard \& Lupton 1998) and implemented into the SNOOPY package. Template subtraction was not applied to all the data as the only template images available, with similar filters to the Landolt system (Johnson-Cousins), were those from the Isaac Newton Telescope described above. The SN photometry was performed relative to a local sequence of 10 stars in the field of NGC 1058, calibrated during several photometric nights by comparison with Landolt standard stars (Fig. 1). The UBVRI magnitudes of the local standards are reported in Table A.1.

In spite of the many different instrumental configurations used, the colour-corrected UBVR photometry of SN $2007 \mathrm{gr}$ appears to be coherent. In contrast, the raw I-band light curve showed a significant scatter (up to $0.4 \mathrm{mag}$ ) due to the differences in the $I$ filter transmission curves between the various instruments. We therefore applied an S-correction to the $I$ band data (Pignata et al. 2008) in order to standardise the photometry. This technique has previously been applied to a number of type Ia SNe (e.g. Stritzinger et al. 2002; Pignata et al. 2004, 2008). Application of the S-correction to the I-band data substantially reduced the scatter in the light curve. The S-corrected light curves of SN 2007gr are displayed in Fig. 2 and the magnitudes are reported in Table A.2.

Near-IR photometry in the $J H K$ bands was obtained on 25 nights over a period of more than one year (Table A.3). The near-IR magnitudes were determined using the same PSF-fitting technique as described for the optical photometry. The SN magnitudes were calibrated with respect to field stars in the $2 \mathrm{MASS}^{2}$ database (see Sect. 4.2 for light curves). The derived JHK magnitudes and their uncertainties are listed in Table A.3. The firstorder colour corrections were omitted when observations in one band only were obtained.

${ }^{2}$ http://www.ipac.caltech.edu/2mass/index.html

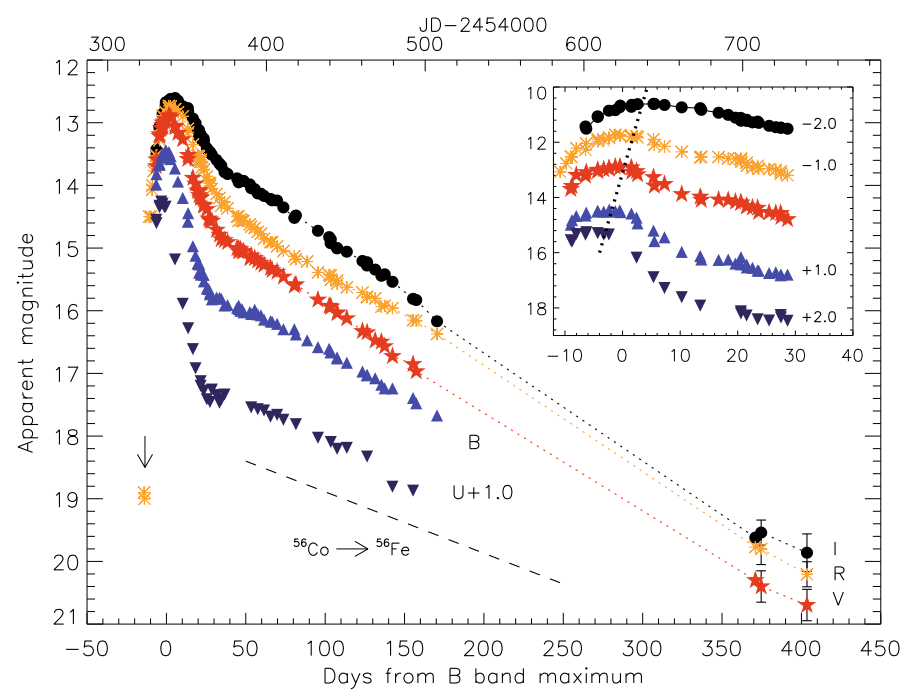

Fig. 2. Complete UBVRI light curves of SN 2007gr. I-band data obtained from the Liverpool and Wendelstein Telescopes have been S-corrected (Sect. 3.1). All errors at phases $<200 \mathrm{~d}$ are smaller than the symbol sizes. The dashed line shows the rate of decay of ${ }^{56} \mathrm{Co} \rightarrow{ }^{56} \mathrm{Fe}$. The arrow indicates the pre-explosion $R$-band detection limit at the location of SN 2007gr. The inset shows the light curve evolution at early times with the dotted line approximately linking the peak magnitudes in all bands.

\subsection{Spectroscopy}

Optical spectra were obtained at 29 epochs, spanning -7.3 to +375.5 days with respect to $B$ maximum. Table A.4 lists the dates, instrument configurations, as well as the resulting spectral resolution and wavelength coverage.

The 2D spectral images were processed in a standard fashion using IRAF, including bias subtraction and flat-field corrections. The spectra were wavelength calibrated using arc lamps; the calibration was checked against night-sky lines and if required small shifts ( $\lesssim$ few $\AA$ ) were applied to the SN spectra. The spectra were flux calibrated using spectrophotometric standardstar spectra taken with an identical instrument configuration. All observations were obtained with a slit angle chosen to minimise the contribution of the two stars flanking SN $2007 \mathrm{gr}$ (see inset Fig. 1). The slit was therefore not necessarily aligned along the parallactic angle. However, the spectra were corrected for light losses by making use of the extensive multi-band photometry available.

Our last optical spectrum was taken on 2008 Sep. 3, approximately 387 days after explosion, with the Gemini Multi-Object Spectrograph on the Gemini North Telescope (GMOS-N). The reduction procedure for this spectrum differed slightly compared to the other spectra as we processed the data with the GMOS pipeline implemented within IRAF. The spectrum revealed significant contamination from nearby bright stars at bluer wavelengths. The spectra of these nearby sources were extracted with the $\mathrm{SN}$ and their flux contributions were subsequently subtracted from the SN spectrum using standard tasks within IRAF. The optical spectra of SN 2007gr are discussed in Sect. 5.1.

SN 2007gr was first observed in the near-IR on 2007 Sep. 9 UT $(+15.5 \mathrm{~d})$, and subsequently ten more times. The bulk of our near-infrared spectroscopic data were acquired using the near-IR imager and spectrometer (NIRI) mounted at the Cassegrain focus of the GEMINI-North Telescope. Three epochs were obtained using the Near Infrared Camera 
Spectrometer (NICS) at the TNG. Details of these observations, including instrument configurations, are given in Table A.5.

The NIRI data were processed using the GEMINI/NIRI pipeline implemented within the IRAF environment, while the TNG data were processed using standard routines in IRAF. All of our near-IR spectra were acquired by nodding the point-source targets along the spectrograph slit in the standard ABBA pattern, facilitating the removal of background emission. For the TNG data, the sky subtraction was performed by subtracting the $A$ frames from the $B$ frames (and vice-versa), and one-dimensional spectra of SN 2007gr were then extracted from the sky-subtracted frames. In the case of the Gemini data, the AB frames were pairwise subtracted and divided by the normalised flat field. A sky frame was then derived for each individual exposure by averaging the nearest 6-10 dithered frames taken within $2 \mathrm{~min}$. At later epochs, the time period was set in such a way so that one sky frame was selected per object image obtained on 2007 Dec. 20 and 2008 Jan. 09.

The near-IR spectra were all wavelength-calibrated using arc lamps. We used spectra of standard stars, taken at similar air masses and on the same nights as the SN spectra, to remove telluric absorptions and to obtain a relative flux calibration. The final flux calibration of the spectra was achieved by applying scaling factors derived from the near-IR photometry (Table A.3). Our series of near-IR spectra are shown in Sect. 5.3.

The spectra presented are in the galaxy rest-frame assuming a redshift of the galaxy NGC 1058 i.e. $z=0.001728^{3}$. From our latest optical spectrum on 2008 Sep. $03(+375.5$ d), we measure a redshift from the $\mathrm{H} \alpha$ line due to NGC 1058 that is consistent with the value reported above. The spectra are corrected for extinction using the standard extinction law of Cardelli et al. (1989) and $E(B-V)_{\text {tot }}=0.092$ mag (see Sect. 4.1).

\section{Photometry}

\subsection{Optical light curves}

The complete optical light curves of SN $2007 \mathrm{gr}$, extending from -6 to $+404 \mathrm{~d}$ after $B$ maximum, are displayed in Fig. 2 . The dense photometric coverage of SN 2007gr allows us to estimate the epoch of maxima and the peak apparent magnitudes in all bands. This was done by fitting each light curve around maximum with a low-order polynomial function. Table 1 lists the peak apparent and absolute magnitudes, as well as the epochs of maxima in all bands with respect to both $B$-band maximum and the date of explosion. All photometry has been corrected for extinction using the relations of Cardelli et al. (1989) and a colour excess $E(B-V)_{\mathrm{tot}}=0.092 \mathrm{mag}$ (see Table 1$)$.

$B$-band maximum occurred on 2007 August 25 at $13.47 \pm$ $0.02 \mathrm{mag}$, with the $V, R$, and $I$ bands peaking respectively about $1.5,4.0$, and $4.0 \mathrm{~d}$ later. With a distance modulus $\mu=29.84$, we estimate the absolute $B$-band magnitude of SN $2007 \mathrm{gr}$ at maximum to be -16.75 . This is the same as for SN 2002ap $\left(M_{B}=-16.76\right)$, but significantly fainter than the GRB associated SN 1998bw $\left(M_{B}=-18.44\right)$ and SN 2003jd $\left(M_{B}=\right.$ $-19.30)^{4}$. Since the peak brightness depends primarily on the mass of ${ }^{56} \mathrm{Ni}$ synthesized in the explosion, we may immediately surmise that SNe 2002ap and 2007gr appear to have comparable quantities of ${ }^{56} \mathrm{Ni}$. We will discuss this further in Sect. 4.4.

\footnotetext{
3 The redshift of NGC 1058 is taken from NED (Nasa/Ipac Extragalactic Database).

${ }^{4}$ References are given in Table 2.
}

The rise time of SN 2007gr, hence likely the time interval between explosion and maximum is constrained by the $R$-band non-detection on 2007 August 10 (Madison \& Li. 2007). This restricts the rise time to $B$ maximum to $11.5 \pm 2.7 \mathrm{~d}$ (i.e. 8.8-14.2 d), indicating that SN 2007gr has an intermediate rise time compared to other type Ic SNe. It is typical to those of SN 2002ap (8 d; Foley et al. 2003), SN 1998bw ( 15 d; Mazzali et al. 2001), and SN 1994I which reached $B$ maximum $\sim 12$ days after explosion (Iwamoto et al. 1994).

We estimated the decline rates for SN $2007 \mathrm{gr}$ by using linear fits to the light curves (see Table 1). The UBVRI light curves reveal a rapid decline after maximum followed by a much slower decline, which is typical of $\mathrm{SNe} \mathrm{Ib} / \mathrm{c}$. The decline rate in the period from 30 to $170 \mathrm{~d}$ after the explosion (i.e. $\sim 0.01-0.017 \mathrm{mag} \mathrm{d}^{-1}$ ) is similar in all optical bands, but typically a bit faster in the redder bands. The decline in the earlier part $(30-100 \mathrm{~d})$ is generally less steep than at later epochs (100-170 d) with the exception of the $R$ band. The $R$ light curve initially declines faster than other optical bands and is followed by a phase of slower decline from 100 to $170 \mathrm{~d}$. This is probably due to the development of forbidden [Ca II] $+[\mathrm{OII}] \sim 7300 \AA$ and [O I] $\lambda \lambda 6300,6364$ arising at $\sim 80 \mathrm{~d}$, as in the case of typical $\mathrm{SNe} \mathrm{Ib} / \mathrm{c}$. At epochs later than 60 days, the light curve slopes of SN 2007gr in all optical bands are found to be steeper than those expected when the energy source is ${ }^{56} \mathrm{Co} \rightarrow{ }^{56} \mathrm{Fe}$ decay and the trapping of $\gamma$-rays is complete $\left[0.98\right.$ mag $\left.(100 \mathrm{~d})^{-1}\right]$ (see Fig. 2). This is probably indicative of significant $\gamma$-ray escape.

In Fig. 3 we compare the UBVRI light curves of SN 2007gr with those of other well observed stripped core-collapse SNe Ic. These $\mathrm{SNe}$ represent an exhaustive sample of type Ic $\mathrm{SNe}$ with well-sampled light curves; luckily, these SNe also span a large range of properties, thereby probing different physical conditions.

The light curves of the $\mathrm{SNe}$ have been scaled in time and magnitude to match the peaks of SN $2007 \mathrm{gr}$ in all filters. Figure 3 illustrates the diversity of SNe Ic in terms of their light curve morphology and decline rates. Although Clocchiatti \& Wheeler (1997) suggest that the decline rates appear to come in slow and fast varieties, new events seem to support the idea that there is a continuous range between these two extremes. Figure 3 shows that after maximum brightness, SN 1994I is the fastest decliner in all filters followed by SN 2003jd, while SN 2004aw is generally the slowest. In the nebular phase, the decline rates $\left(\gamma_{\mathrm{LC}}\right)$ of the light curves are determined by the energy deposition of the $\gamma$-rays produced in the ${ }^{56} \mathrm{Ni} \rightarrow{ }^{56} \mathrm{Co} \rightarrow{ }^{56} \mathrm{Fe}$ radioactive decay chain. Thus the emitted luminosity includes the $\gamma$-ray contributions which result from the energy deposition in the ${ }^{56} \mathrm{Co}$ decay and from the annihilation of electron-positron pairs, in which the kinetic energy of the positrons is an important factor. Assuming homologous expansion and the incomplete trapping of $\gamma$-rays and positrons, the behaviour of the light curve depends on the mass ejected $\left(M_{\mathrm{ej}}\right)$ and the kinetic energy $\left(E_{\mathrm{kin}}\right)$ according to the following relation (see Valenti et al. (2008b) and references therein for a simple derivation),

$\gamma_{\mathrm{LC}} \propto M_{\mathrm{ej}} / E_{\mathrm{kin}}{ }^{1 / 2}$.

Hence, fast decliners such as SN 1994I $\left(\gamma_{\mathrm{LC}} \sim 0.88\right)$ are typical of SNe with lower ratios of ejecta mass to kinetic energy than the slow decliners of SN 2004aw $\left(\gamma_{\mathrm{LC}} \sim 2.3\right)$ and SN 1998bw $\left(\gamma_{\mathrm{LC}} \sim 1.8\right)$ (see Table 2). Meanwhile, the $U$ band of SN $2007 \mathrm{gr}$ fades at a rate similar to those of SNe 2004aw and 2002ap from $B$ maximum to $\sim 50 \mathrm{~d}$ later, while the later evolution is slower than in SN 2002ap. For the $B, V$ and $R$ bands, the decline is very similar to SNe 2002ap and 1998bw, and faster than SN 2004aw. 
Table 1. The photometric parameters of SN 2007gr.

\begin{tabular}{|c|c|c|c|c|c|c|c|c|}
\hline & $U$ & $B$ & $V$ & $R$ & $I$ & $J$ & $H$ & $K$ \\
\hline $\begin{array}{l}\text { Apparent mag. } \\
\text { at maximum }\end{array}$ & $13.29 \pm 0.02$ & $13.47 \pm 0.02$ & $12.91 \pm 0.01$ & $12.76 \pm 0.02$ & $12.61 \pm 0.02$ & $12.40 \pm 0.18$ & $12.06 \pm 0.10$ & $11.66 \pm 0.10$ \\
\hline $\begin{array}{l}\text { Absolute mag. } \\
\text { at maximum }{ }^{a}\end{array}$ & $-16.99 \pm 0.19$ & $-16.75 \pm 0.19$ & $-17.22 \pm 0.18$ & $-17.29 \pm 0.18$ & $-17.36 \pm 0.17$ & $-17.52 \pm 0.20$ & $-17.83 \pm 0.20$ & $-18.21 \pm 0.20$ \\
\hline Extinction (mag) & $0.44 \pm 0.09$ & $0.38 \pm 0.08$ & $0.29 \pm 0.06$ & $0.21 \pm 0.04$ & $0.13 \pm 0.03$ & $0.08 \pm 0.02$ & $0.05 \pm 0.01$ & $0.03 \pm 0.01$ \\
\hline $\begin{array}{l}\text { JD of } \\
\operatorname{maximum}^{b}\end{array}$ & $54334.8 \pm 0.8$ & $54337.0 \pm 1.0$ & $54338.5 \pm 1.1$ & $54341.0 \pm 1.7$ & $54341.0 \pm 1.5$ & $54341.4 \pm 2.3$ & $54342.4 \pm 4.3$ & $54342.3 \pm 3.5$ \\
\hline $\begin{array}{l}\text { Epoch of } \\
\text { maximum (days) }\end{array}$ & $-2.2 \pm 1.3$ & $0 \pm 1.0$ & $1.5 \pm 1.5$ & $4.0 \pm 2.0$ & $4.0 \pm 1.8$ & $4.4 \pm 2.5$ & $5.4 \pm 4.4$ & $5.3 \pm 3.6$ \\
\hline $\begin{array}{l}\text { Rising } \\
\text { times (days) }\end{array}$ & $9.3 \pm 2.8$ & $11.5 \pm 2.7$ & $13.0 \pm 2.9$ & $15.5 \pm 3.2$ & $15.5 \pm 3.1$ & $15.9 \pm 3.0$ & $16.9 \pm 5.1$ & $16.8 \pm 4.4$ \\
\hline
\end{tabular}

\section{Decline rates ${ }^{e}$}

$(30-100 \mathrm{~d})^{c}$

$(100-170 \mathrm{~d})^{c}$

$(30-170 \mathrm{~d})^{c}$
$0.0095 \pm 0.00070 .0111 \pm 0.00060 .0170 \pm 0.00050 .0194 \pm 0.00070 .0164 \pm 0.00040 .0304 \pm 0.00060 .0268 \pm 0.00080 .0342 \pm 0.0008$

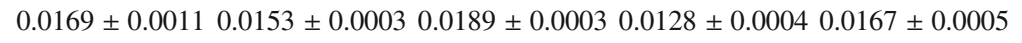
$0.0114 \pm 0.00060 .0130 \pm 0.00040 .0171 \pm 0.00030 .0159 \pm 0.00050 .0169 \pm 0.0002$

${ }^{a}$ A distance modulus $\mu=29.84 \pm 0.16 \mathrm{mag}$ and a colour excess $E(B-V)=0.092$ mag were adopted. ${ }^{b}$ The uncertainty in the epoch of maximum is defined as the time period in which the magnitude is not more than 0.01 mag fainter than the peak magnitude (Yoshii et al. 2003). ${ }^{c}$ Epoch with respect to the estimated $B$-band maximum JD $2454337.0 \pm 1.0{ }^{d}$ Epoch with respect to the estimated explosion date JD $2454325.5 \pm 2.5$.

${ }^{e}$ Decline rates $\left(\mathrm{mag} \mathrm{d}^{-1}\right)$ were estimated using linear least-square fitting.

Table 2. The parameters of type Ic SNe.

\begin{tabular}{|c|c|c|c|c|c|c|c|c|c|c|}
\hline $\mathrm{SN}$ (Ic) & $\begin{array}{l}{ }^{56} \mathrm{Ni} \text { mass } \\
\left(M_{\odot}\right)\end{array}$ & $\begin{array}{l}\text { Ejecta mass } \\
\left(M_{\odot}\right)\end{array}$ & $\begin{array}{l}E_{\text {kin }} \\
\left(10^{51} \mathrm{erg}\right)\end{array}$ & $m_{B, \max }$ & $M_{B, \max }{ }^{a}$ & $\begin{array}{l}\text { Rise } \\
\text { times (d) }\end{array}$ & $E(B-V)^{b}$ & $\mu$ & Redshift $^{c}$ & Ref. \\
\hline $2007 \mathrm{gr}$ & $0.076 \pm 0.020$ & $2.0-3.5$ & $1-4$ & 13.47 & -16.75 & $11.5 \pm 2.5$ & 0.092 & 29.84 & 0.001728 & 1,2 \\
\hline 2004aw & $0.30 \pm 0.05$ & $3.5-8.0$ & $3.5-9.0$ & 18.06 & -17.63 & $\ldots$ & 0.370 & 34.17 & 0.015914 & 3 \\
\hline 2003jd & $0.36 \pm 0.04$ & $2.5-3.5$ & $5-10$ & 15.75 & -19.30 & $\sim 13$ & 0.144 & 34.46 & 0.018860 & 4 \\
\hline 2002 ap & $0.09,0.07$ & $2.5-5.0$ & $4-10$ & 13.11 & -16.76 & $\sim 8$ & 0.090 & 29.50 & 0.002192 & $5,6,7,8,9$ \\
\hline 1998bw & $0.7,0.4,0.5$ & 10.9 & $20-50$ & 14.09 & -18.44 & $\sim 15$ & 0.060 & 32.28 & 0.008670 & $10,11,12,13$ \\
\hline $1997 \mathrm{ef}$ & 0.13 & 9.6 & 17.5 & 17.45 & -16.18 & $\ldots$ & 0.000 & 33.63 & 0.011688 & 14 \\
\hline 1994I & 0.07 & 0.88 & 1 & 13.77 & -17.06 & $\sim 12$ & 0.300 & 29.60 & 0.001544 & $15,16,17$ \\
\hline
\end{tabular}

References: ${ }^{1}$ Valenti et al. (2008a); ${ }^{2}$ this paper; ${ }^{3}$ Taubenberger et al. (2006); ${ }^{4}$ Valenti et al. (2008b); ${ }^{5}$ Yoshii et al. (2003); ${ }^{6}$ Foley et al. (2003);

${ }^{7}$ Gal-Yam et al. (2002); ${ }^{8}$ Tomita et al. (2006); ${ }^{9}$ Mazzali et al. (2002); ${ }^{10}$ Iwamoto et al. (1998); ${ }^{11}$ Galama et al. (1998); ${ }^{12}$ Nakamura et al. (2001);

${ }^{13}$ Patat et al. (2001); ${ }^{14}$ Mazzali et al. (2000); ${ }^{15}$ Nomoto et al. (1994); ${ }^{16}$ Richmond et al. (1996); ${ }^{17}$ Sauer et al. (2006). ${ }^{a}$ The peak absolute magnitudes were estimated using the apparent magnitudes at maxima. ${ }^{b}$ Total extinction. ${ }^{c}$ Redshift of the host galaxy (taken from the Nasa/IPAC Extragalactic Database NED).

\subsection{Near-IR light curves}

Our near-IR photometry for SN 2007gr is reported in Table A.3. Early observations in the $J$-band enable us to constrain the peak magnitude and epoch of $J$ maximum. This is likely to have occurred on 2007 August 29, 16 days after the explosion date and 4.4 days after $B$ maximum.

In Fig. 4 we compare the $J H K$ lightcurves of SN $2007 \mathrm{gr}$ with those of SN 2002ap (Tomita et al. 2006), which has the best coverage of all SNe Ic at near-IR wavelengths. In the nearIR, the light curves of SN $2007 \mathrm{gr}$ are very similar to those of SN 2002ap in terms of light curve morphology. Approximate peak magnitudes have been derived using the additional coverage of SN 2002ap as a template, particularly for the $H$ and $K$ bands (see Table 2) where the rising branch is unconstrained. The estimated peaks of SN 2007gr suggest that the time delay between $B$ and $J$ maximum is comparable to SN 2002ap ( $\sim 5$ days for SN 2007gr and 7 days for SN 2002ap). The estimated decline rates for the period from +30 to $+140 \mathrm{~d}$ after $B$ maximum in the $J, H$ and $K$ bands are $0.029,0.024$ and $0.026 \mathrm{mag} \mathrm{d}^{-1}$, respectively, again similar to those of SN 2002ap. However, the steeper decline rates of SN 2002ap at later epochs $(>+120 \mathrm{~d})$ indicate a greater $\gamma$-ray leakage from the radioactive decay than for SN $2007 \mathrm{gr}$.

\subsection{Colour evolution of SN $2007 \mathrm{gr}$}

The colour curves of SN 2007gr were produced using the optical and near-IR photometry detailed above. In Fig. 5, we present the $U-B, B-V, V-R, V-J, V-K$ and $H-K$ colour curves of SN $2007 \mathrm{gr}$ and comparisons with the same sample of type Ic SNe: 2004aw, 2003jd, 2002ap, 1998bw and 1994I. All curves have been corrected for reddening using the relations given by Cardelli et al. (1989) (see Table 2 for values and references). 

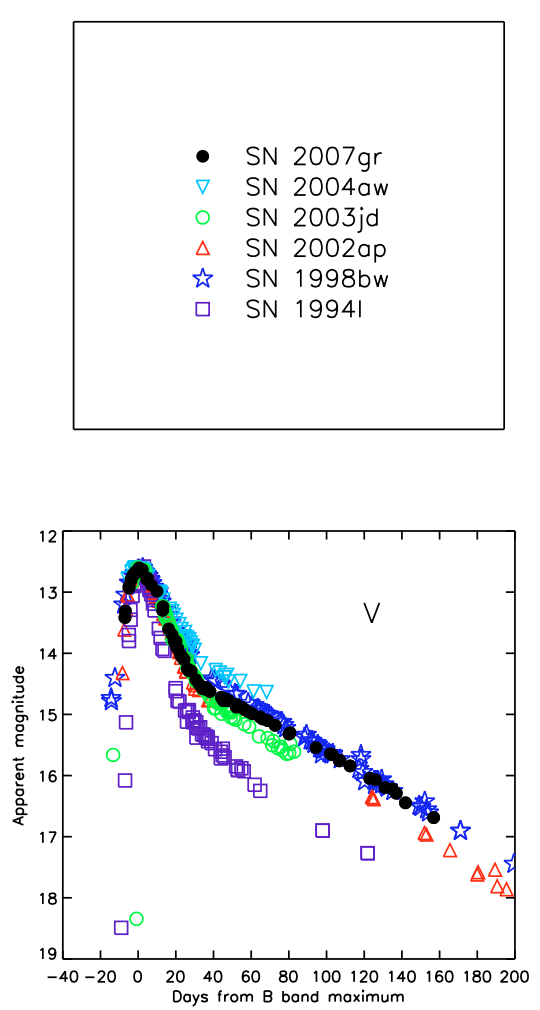
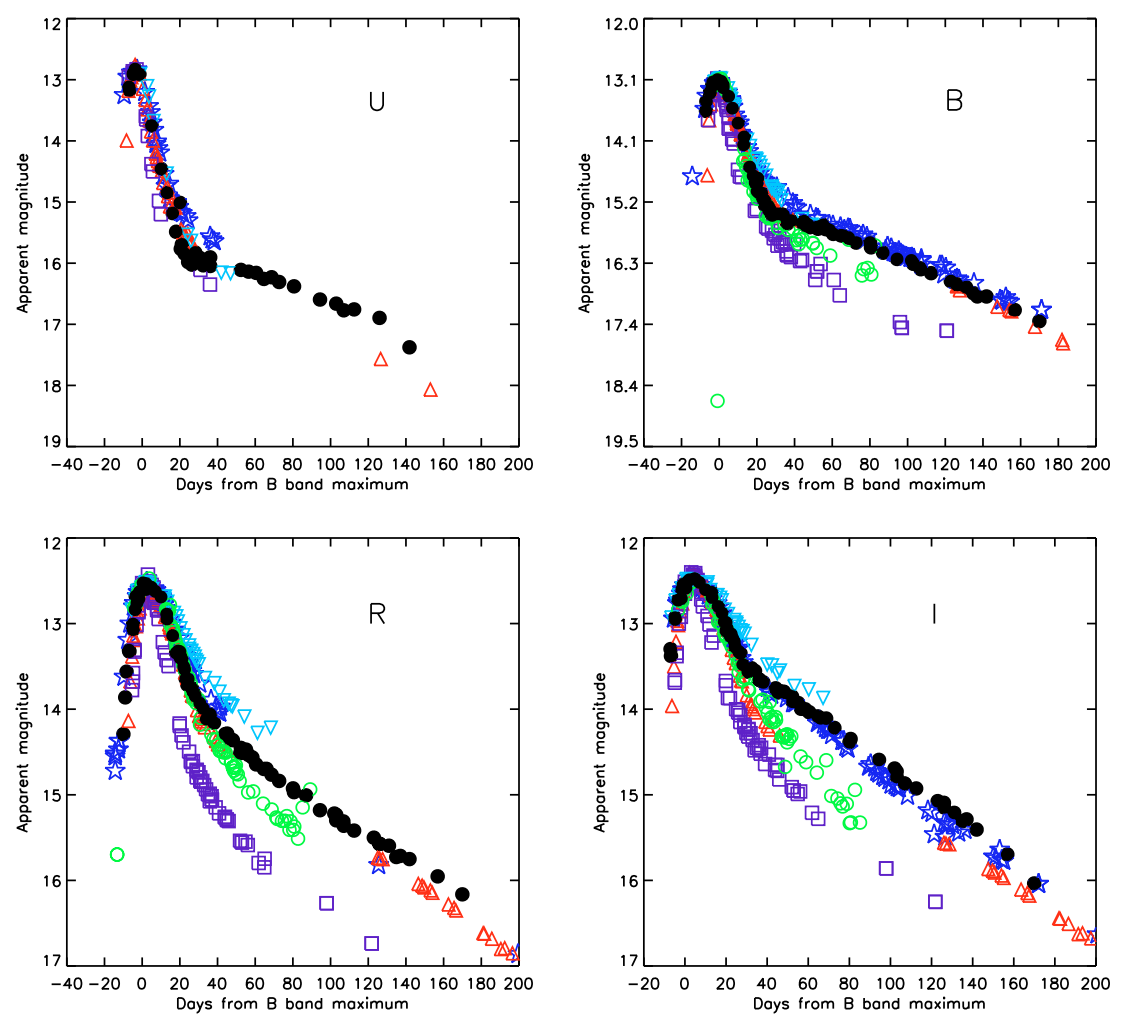

Fig. 3. Optical light curves of SN 2007gr compared to those of the type Ic SNe 2004aw (Taubenberger et al. 2006), 2003jd (Valenti et al. 2008b), 2002ap (Pandey et al. 2003; Foley et al. 2003; Tomita et al. 2006), 1998bw (Galama et al. 1998; McKenzie \& Schaefer 1999) and 1994I (Yokoo et al. 1994; Gyoon et al. 1995; Lee et al. 1995; Tsvetkov \& Pavlyuk 1995; Richmond et al. 1996). All the light curves presented have been shifted in time and magnitude to match SN $2007 \mathrm{gr}$ at maximum.

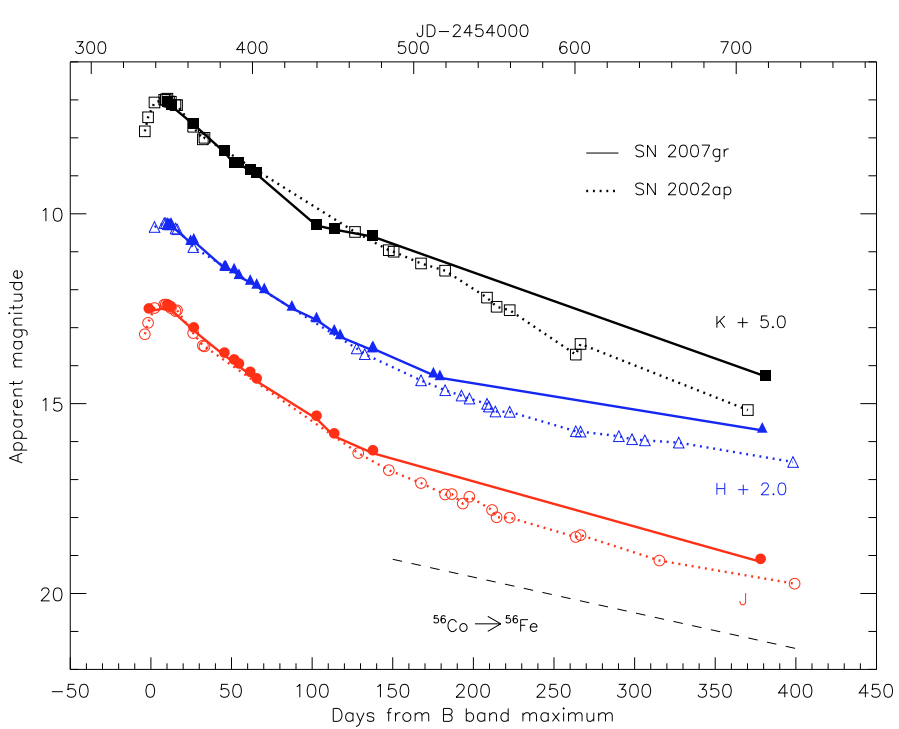

Fig. 4. The near-IR light curves of SNe 2007gr and 2002ap (Tomita et al. 2006). The filled symbols joined by the full lines represent the data for SN 2007gr. The data for SN 2002ap (open symbols, dotted lines) have been shifted in magnitude by $0.7,0.7$ and 0.5 mag to match SN $2007 \mathrm{gr}$ at $\sim 20 \mathrm{~d}$ in the $J H K$ bands, respectively.

The colour evolution of all SNe presented become monotonically redder from $B$ maximum to $\sim+20 \mathrm{~d}$. After this time, the differences between the $\mathrm{SNe}$ become evident.

The early $U-B$ colour of SN 2007gr (Fig. 5, top-left panel) shows a steep rise by $\sim 1.5 \mathrm{mag}$. This increasingly redder colour may be due to both the cooling of the continuum and the progressive increase of the metal line blanketing in the $U$ band. After the peak, the $U-B$ colour of SN 2007gr gradually turns bluer, followed by a plateau for $\sim 60 \mathrm{~d}$. Intermediate-phase data for SN 2002ap are lacking, but at $t=150$ days it appears to have reddened considerably by $\sim 0.3$ mag with respect to the colour at $\sim+20$ d. SN $2007 \mathrm{gr}$ also marginally reddens around this epoch.

The $B-V$ colour indices of all $\mathrm{SNe}$ (Fig. 5, top-middle panel) in this sample rise at an approximate linear rate for the first 3 weeks before the $B$ band maximum. After the colour peak, they rapidly become bluer, except for SN 2004aw which remains the reddest at $1.2 \mathrm{mag}(\sim+50 \mathrm{~d})$ before showing any sign of a decline. In the case of SN 2004aw, it is difficult to ascertain whether this behaviour is intrinsic to the SN, or simply due to an underestimate of the reddening as has already been suggested by Benetti et al. (2006). On the contrary, SN 1994I remains the bluest SN ( $B-V \sim 0.2-0.5 \mathrm{mag}$ at $\sim+50 \mathrm{~d}$ ) throughout its evolution. The $B-V$ colour of SN $2007 \mathrm{gr}$ is intermediate between these two objects, with strong similarity to SNe 1998bw and 2002ap. Uncertainties in the reddening estimates and possible contamination from nearby blue stars preclude a further discussion of the bluer colours of SN 1994I (Taubenberger et al. 2006).

The early $V-R$ colour evolution $(<+80 \mathrm{~d})$ of SN $2007 \mathrm{gr}$ is comparable to the evolution of $\mathrm{SN} 2004 \mathrm{aw}$ with a fast rise to maximum at $V-R \sim 0.5 \mathrm{mag}$ after $\sim+20 \mathrm{~d}$. Following the peak, all $\mathrm{SNe}$ tend to become bluer for a short period until $\sim+70 \mathrm{~d}$. This can be explained in part by cooling due to ejecta expansion, with the peak of the spectral energy distribution shifting towards longer wavelengths. After this period, SN 2007gr gradually reddens, following the evolution of SN 2002ap which reaches a $V-R$ colour of $\sim 1.3 \mathrm{mag}$ at $\sim+300 \mathrm{~d}$. The onset of this reddening is consistent with the development of the forbidden 

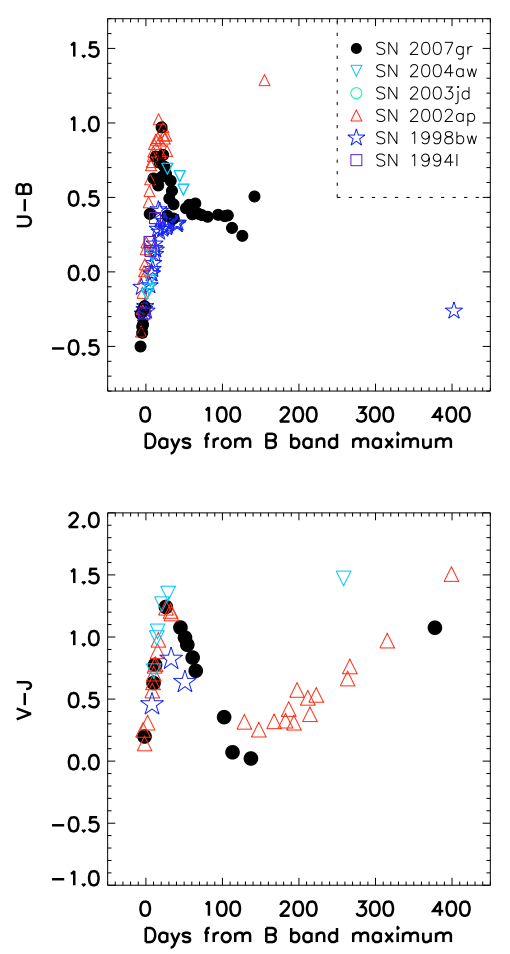
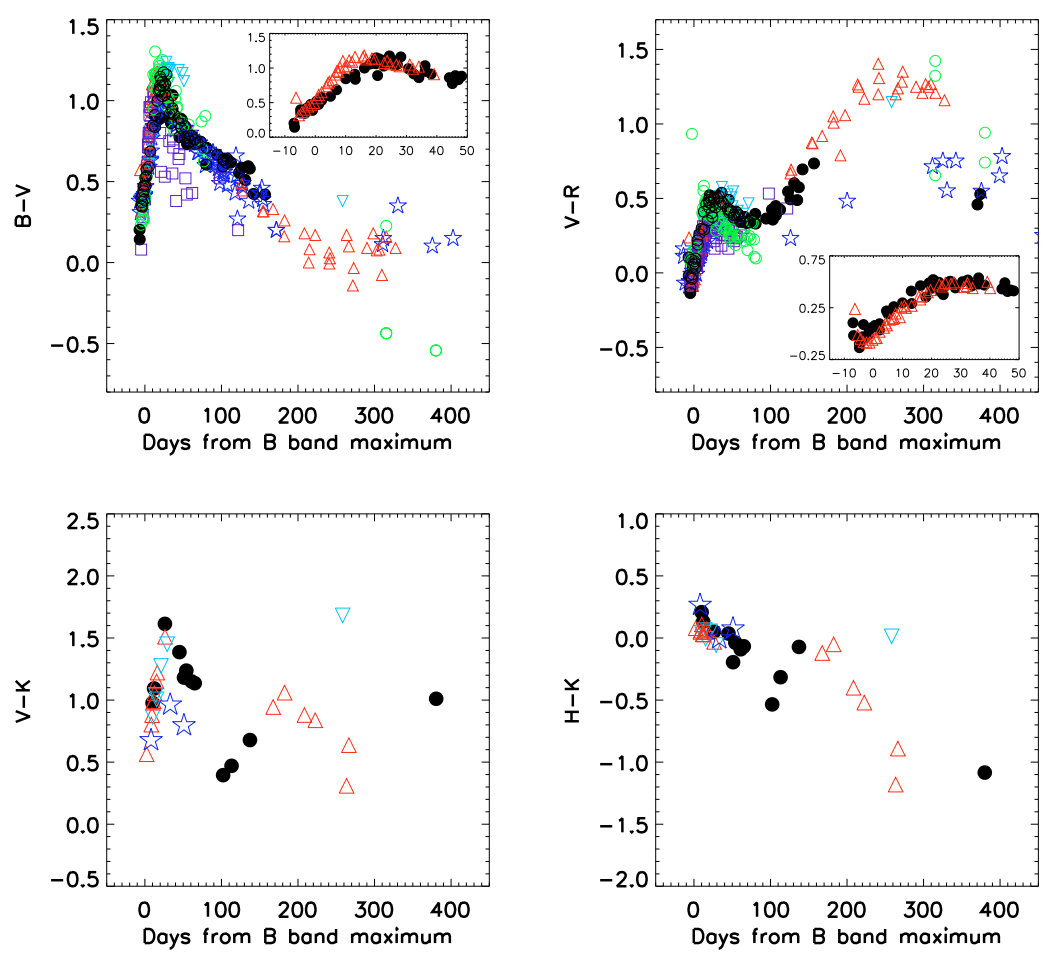

Fig. 5. Optical and near-infrared colour evolution of SN 2007gr compared with other SNe Ic: SN 2002ap, SN 2004aw, SN 1998bw, SN 1994I. Evolution of the $U-B$ (top-left), $B-V$ (top-middle), $V-R$ (top-right), $V-J$ (bottom-left), $V-K$ (bottom-middle) and $H-K$ (bottom-right) colours. The insets in two of the upper panels show a zoom-in of the early colour evolution of SN 2007gr compared to SN 2002ap. All curves have been corrected for reddening. The Cardelli et al. (1989) law was used to estimate the extinction in the different bands. See text for references.

[O I] $\lambda \lambda$ 6300, 6364 emission and the blend of [Ca II] $\lambda \lambda 7291$, 7323 and $[\mathrm{O}$ II] $\lambda \lambda$ 7320, 7330 (see Fig. 7).

In Fig. 5, the $V-J$ and $V-K$ colour curves of all SNe become monotonically redder, reaching a peak at $\sim+30 \mathrm{~d}$. This is then followed by a rapid decline to the blue up to $+150 \mathrm{~d}$ before undergoing a further transition to a redder colour. In the near-IR too, the colour evolution of SN $2007 \mathrm{gr}$ best resembles SN 2002ap. The $V-J$ colours of SNe 2007gr and 2002ap redden to $\sim 1.5 \mathrm{mag}$ at $\sim+400 \mathrm{~d}$.

Signatures of any dust that might have formed in the ejecta of SN 2007gr should be evident in the near-IR colour evolution at late times. Furthermore, the $H-K$ colour evolution tracks the evolution of the emission due to the first overtone of the carbonmonoxide molecule at $>2.3 \mu \mathrm{m}$ (see Fig. 16 Sect. 5.5), which may be a precursor to dust condensation in the ejecta. Indeed, the $H-K$ colour curve of SN 2007gr tallies with the appearance and disappearance of the CO-feature between $\sim+70$ to +200 days relative to $B$ maximum. Such an obvious signature of $\mathrm{CO}$ is not apparent in the $H-K$ colour curves of any of the other Ic SNe shown in Fig. 5, with the possible exception of SN 1998bw which shows a mild upturn at about $+60 \mathrm{~d}$, although the near-IR coverage for all objects other than SN $2007 \mathrm{gr}$ is admittedly lacking at epochs of interest. For SN $2007 \mathrm{gr}$, given the relatively blue $H-K$ colour $\left(E_{H-K}\right.$ is $\left.\sim-1.2 \mathrm{mag}\right)$ at about $+400 \mathrm{~d}$, we conclude that dust formation is unlikely to have occured in SN $2007 \mathrm{gr}$ by this epoch.

\subsection{The bolometric (uvoir) light curves and physical parameters}

We constructed an optical near-infraRed (UVOIR) bolometric light curve by integrating the UBVRIJHK broad band fluxes. To derive the integrated SN luminosity, the magnitudes were corrected for reddening, converted to a flux density at the effective wavelength, and finally integrated using Simpson's rule. The integrated flux was then converted to a luminosity using the distance modulus as assumed in Sect. 2 i.e. $\mu=29.84 \pm 0.16$.

The bolometric light curve of SN $2007 \mathrm{gr}$ is shown in Fig. 6. For comparison, we have also included the bolometric light curves of the SNe Ic sample. Following Valenti et al. (2008a), we applied a toy-model to the bolometric light curve of SN 2007gr in order to constrain the physical parameters of the explosion (see Table 2). These include the ejected mass $\left(M_{\mathrm{ej}}\right)$, the nickel mass $\left(M_{56} \mathrm{Ni}\right)$, and the kinetic energy $\left(E_{\mathrm{kin}}\right)$. The procedure is based on a two-component analytical model to account for the photospheric and nebular phases of expansion. During the photospheric phase ( $t \leq 30 \mathrm{~d}$ past explosion) homologous expansion of the ejecta, spherical symmetry, and the location of the radioactive ${ }^{56} \mathrm{Ni}$ exclusively in the core is assumed (Arnett et al. 1982). At late times ( $t \geq 60 \mathrm{~d}$ past explosion), the model includes the energy contribution from the ${ }^{56} \mathrm{Ni} \rightarrow{ }^{56} \mathrm{Co} \rightarrow{ }^{56} \mathrm{Fe}$ decay, following the work of Sutherland \& Wheeler (1984) and Cappellaro et al. (1997). In the nebular phase the ejecta are optically thin, so that the incomplete trapping of $\gamma$-rays and positrons has to be accounted for (Clocchiatti \& Wheeler 1997).

The modelling of the bolometric light curve suggests that the ejected mass $M_{\mathrm{ej}}$ of SN $2007 \mathrm{gr}\left(2-3.5 M_{\odot}\right)$ is similar to SN 2002ap but the kinetic energy $E_{\text {kin }}$ is considerably lower (by a factor of $\sim 3$ ). However, we do note that the parameters for SN 2002ap (see Table 2) have been derived via a more involved modelling procedure (e.g. Mazzali et al. 2002; Foley et al. 2003).

A comparison of the early quasi-bolometric light curves shows that SN 2007gr appears to be most similar to SN 2002ap in terms of peak luminosity and width. As alluded to previously, the similarity in peak luminosity $\left(M_{\mathrm{Bol}}=-16.9\right)$ implies that these $\mathrm{SNe}$ produced similar quantities of ${ }^{56} \mathrm{Ni}\left(\sim 0.07-0.1 M_{\odot}\right)$. 


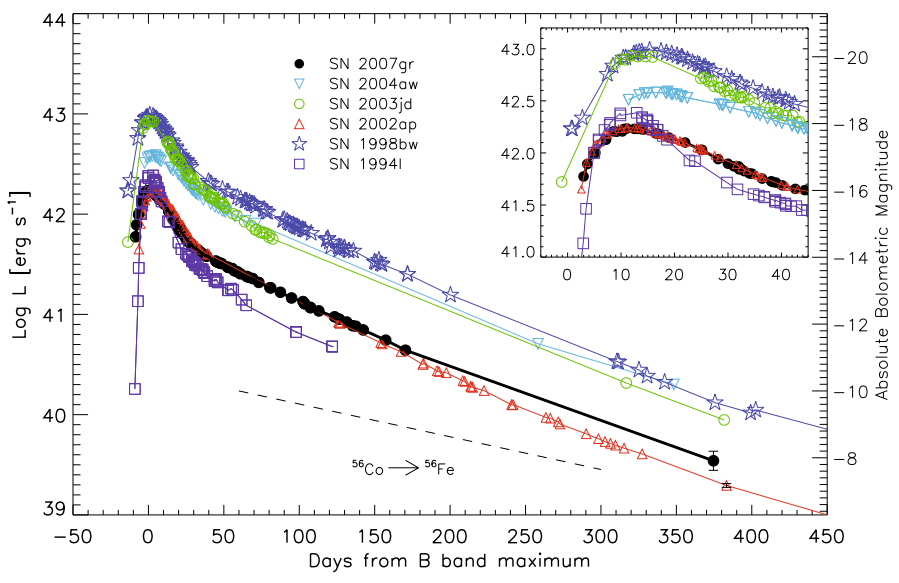

Fig. 6. The uvoir bolometric light curve of SN 2007gr and other type Ic SNe. The inset shows the differences in the widths of the uvoir lightcurves of this sample of type Ic SNe.

We estimate that the quantity of ${ }^{56} \mathrm{Ni}$ ejected in SN $2007 \mathrm{gr}$ is $0.076 \pm 0.020$, similar to SNe 2002ap and 1994I. Interestingly, SN 2007gr also resembles SN 2003jd in the shape of the early bolometric light curve, although at a much lower luminosity, while the rise to peak luminosity is comparable to SN 1994I. The rise time of stripped envelope SNe depends both on the radius of the progenitor prior to explosion and on the quantity of ejected mass (Arnett 1996). For reference, we list the rise times of a sample of $\mathrm{Ib} / \mathrm{c} \mathrm{SNe}$ in Table 2 collected from the literature, but note that for all objects other than SN $2007 \mathrm{gr}$, these are observationally poorly constrained, and the reality of any differences in the rise times cannot at this point be directly attributed to physical differences between the progenitor stars.

SNe $2007 \mathrm{gr}$ and 2002ap have similar peak widths which are evidently broader than that of SN 1994I (see Fig. 6 inset). Such broad peaks are usually attributed to more massive SNe Ic progenitors (e.g. Arnett et al. 1982; Mazzali et al. 2001; Foley et al. 2003). Since the peak widths indicate the rate of energy diffusion out of the core, this suggests that the progenitors of SNe $2007 \mathrm{gr}$ and 2002ap had relatively large masses. We also expect the mass ejected in these two events to be larger compared to SN 1994I but less than for SNe 1998bw and 2004aw.

The bolometric light curve of SN $2007 \mathrm{gr}$ indicates a latetime (120 to 380 day) decline of $\gamma=0.0142 \pm 0.0002 \mathrm{mag} \mathrm{d}^{-1}$. These decline rates are comparable to those of the broad-lined SNe 1998bw and 2002ap which are $\gamma=0.0156 \pm 0.0001 \mathrm{mag} \mathrm{d}^{-1}$ and $\gamma=0.0162 \pm 0.0003 \mathrm{mag} \mathrm{d}^{-1}$, respectively. The decline rates are faster than expected from the decay of radioactive ${ }^{56} \mathrm{Co}$ (i.e. $0.0098 \mathrm{mag} \mathrm{d}^{-1}$ ). If the decay of ${ }^{56} \mathrm{Co}$ is responsible for powering the late-time light curve of SN $2007 \mathrm{gr}$, then this suggests that the steeper decline is due to a decreasing $\gamma$-ray trapping in the SN envelope with time.

In Table 2, we compare SN 2007gr to other type Ic SNe which show a wide range of physical properties at the explosion epoch. We can see that SN 2007gr is most similar to SNe 2002ap and $1994 \mathrm{I}$ in terms of the amount of ${ }^{56} \mathrm{Ni}$ synthesised. It can also be noted that SN 2007gr appears to be similar to SN 1994I with respect to the ratio of kinetic energy to ejected mass. Thus SN 2007gr may be considered as the link between broad-lined events like SN 2002ap and low energy events like SN 1994I. We note however, that the parameters listed in Table 2 have been derived using a variety of methods.

\section{Spectroscopy}

\subsection{Optical spectra}

The spectroscopic evolution of SN $2007 \mathrm{gr}$, from 7.3 days prior to maximum light to +375.5 days after, is densely covered and is shown in Fig. 7. The line identifications are based on those by Valenti et al. (2008a).

The early spectra exhibit $\mathrm{Ca}$ II $\mathrm{H} \& \mathrm{~K}, \mathrm{Mg}$ II $\lambda$ 4481, and $\mathrm{Na}$ I D $\lambda \lambda$ 5891, 5897 which are responsible for the P-Cygni absorption features observed at 3810-3850 $\mathrm{A}, 4390-4490 \AA$, and 5720-5760 ̊, respectively. The Si II $6355 \AA$ absorption can also be identified in the early spectra, however it fades around two weeks after maximum light.

Chornock et al. (2007) classified SN 2007gr as a type Ib/c from a spectrum obtained the night following discovery while waiting for follow-up observations. Eventually, no strong evidence for 6678 and $7065 \AA$ He features which are typically in type Ib $\mathrm{SNe} \sim 7$ days after maximum light were found. Consequently, SN 2007 gr was classified as a type Ic. Valenti et al. (2008a) studied the feature at $6450 \AA$, which may be misidentified as $6678 \AA \mathrm{He}$ I in early-time spectra, and suggest that a more plausible identification is C II $6580 \AA$ at a velocity of $\sim 11000 \mathrm{~km} \mathrm{~s}^{-1}$. Indeed this feature disappears around maximum together with another feature at $7000 \AA$ attributable to C II $7235 \AA$, thereby providing support for the Ic classification.

Moreover, almost two weeks after $B$ maximum, one of the strongest optical transitions of the C I ion (9095 $\AA$ ) appears, reaching velocities of $\sim 8000 \mathrm{~km} \mathrm{~s}^{-1}$. The spectrum at around three weeks display two additional C I absorptions (9094, $9406 \AA$ ) due to the extended coverage at longer wavelengths (Valenti et al. 2008a) (see Sect. 5.2 and for further discussions).

The first ten spectra, extending from one week before $B$ maximum to one week after, are characterised by an initially blue continuum which reddens significantly with time. Following maximum light, the Ca II near-IR triplet and the Na I D lines become very prominent in the spectra. Other absorption features include Fe II (4924, 5018, $5169 \AA$ ) and Sc II (5552 $)$. Approximately one week after maximum, the absorption troughs centred at $4200 \AA$ and $4800 \AA$ show the $W$-shaped profile that is characteristic of many type Ic $\mathrm{SNe}$ at a comparable epoch. By this time, the flux in the bluer bands is significantly reduced which is consistent with the redder $U-B$ and $B-V$ colours (Fig. 5).

The spectral evolution of SN 2007gr is relatively fast with the disappearance of Si II $6355 \AA$ already two weeks after maximum. The relatively early appearance of $[\mathrm{CaII}]+[\mathrm{OII}] \sim$ $7300 \AA$ and [O I] $\lambda \lambda 6300,6364$ at about $+55 \mathrm{~d}$ represents the onset of the nebular phase. The absorption features in the spectroscopic evolution of SN $2007 \mathrm{gr}$ tend to drift towards the red with time, as expected. This is a result of the formation of lines occuring in slower, deeper layers of the ejected material. The late-time spectra are dominated by the emission of forbidden lines such as Mg I] 4571 А, [O I] $\lambda \lambda$ 6300, 6364, [Ca II] $\lambda \lambda$ 7291, 7323, and the permitted transitions of $\mathrm{NaI} \mathrm{D}$, O I 7774, and the possible blend of C I ( $8727 \AA)$ and the Ca II near-IR triplet.

The optical spectra do not reveal any clear indication for the presence of He I lines. If He I 5876 $\AA$ was present it would almost certainly be contaminated by $\mathrm{Na}$ I D. Other lines of He I in this region (e.g. 4471, $7065 \AA$ ) would also be blended with other strong features at these wavelengths. Since the optical spectra alone are insufficient to detect small amounts of He I, in Sect. 5.3 we examine the near-IR spectra of SN $2007 \mathrm{gr}$. The He I $10830 \AA$ 


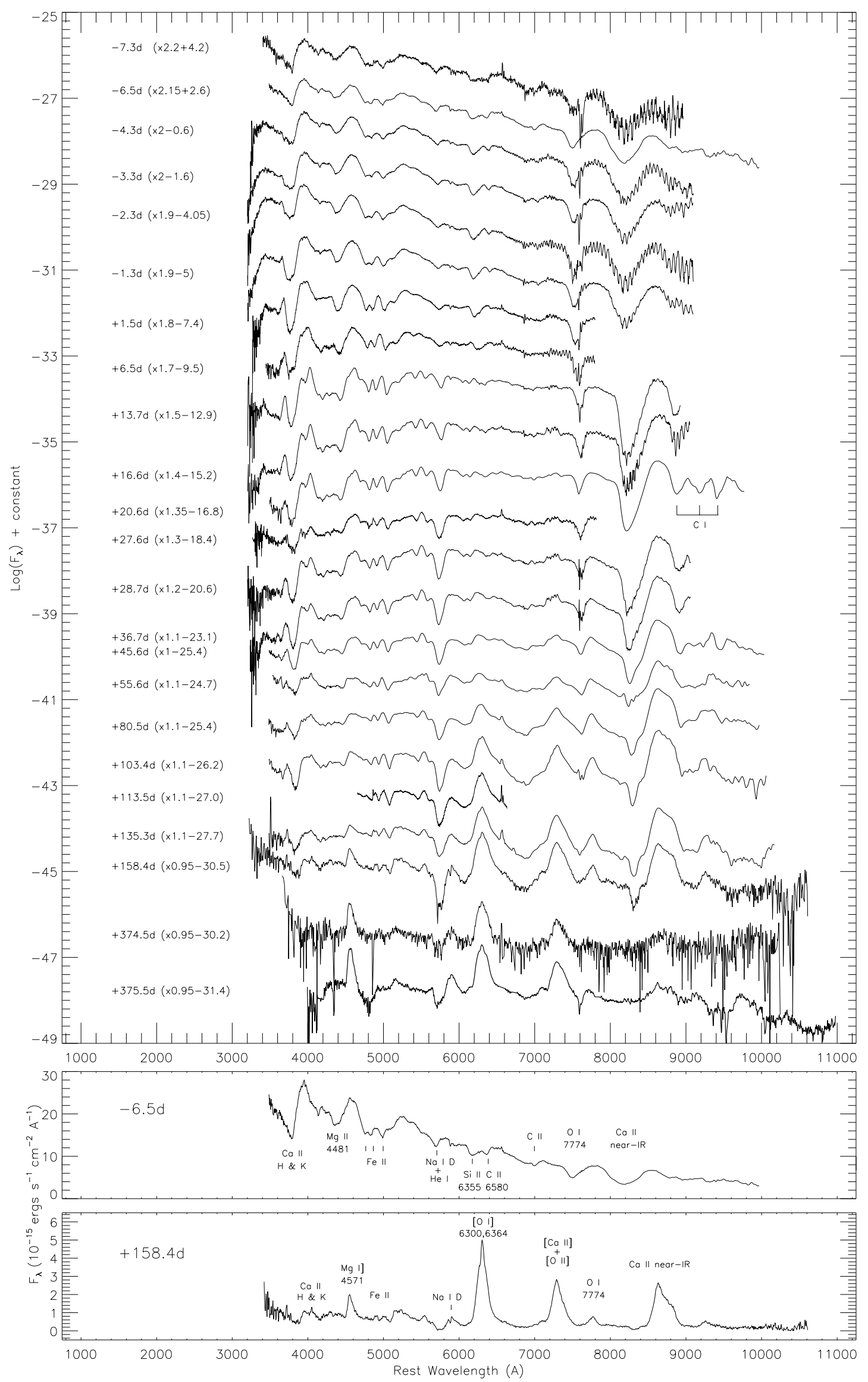

Fig. 7. Evolution of the optical spectra of SN $2007 \mathrm{gr}$. The spectra have been corrected for reddening, $E(B-V)_{\text {tot }}=0.092$, and for the host galaxy redshift of NGC 1058, $z=0.001728$. The spectra have also been magnified and displaced vertically for clarity by the numbers shown in brackets. Identifications are shown in the spectra of SN $2007 \mathrm{gr}$ at -6.5 and +158.4 days relative to $B$ maximum. 


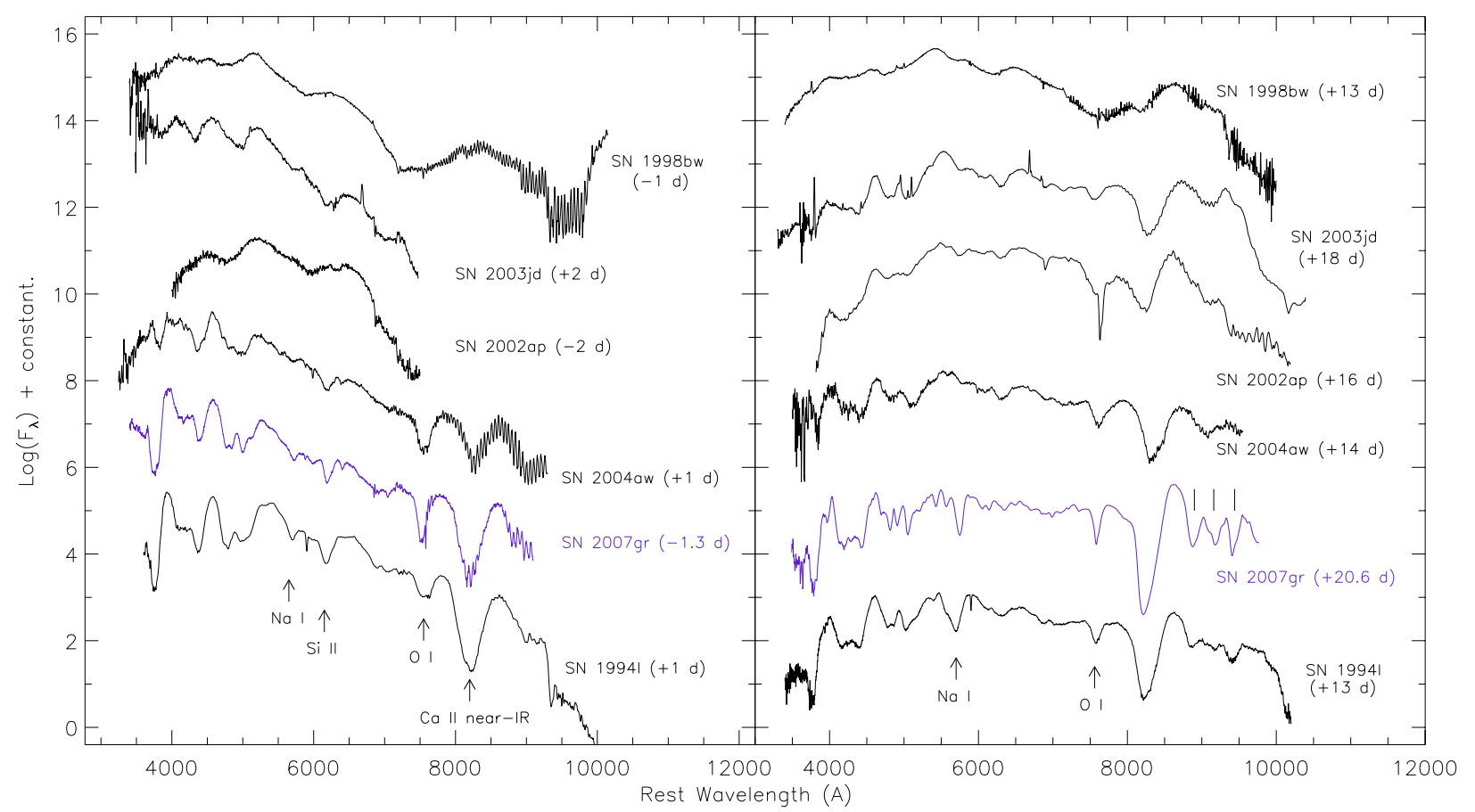

Fig. 8. Comparison of spectra of SNe close to $B$-band maximum (left $)$ and two-three weeks post maximum light (right). All spectra are de-reddened and redshifted assuming the values reported in Table 2. Typical absorption lines are identified. The three solid lines in the right-hand panel next to SN 2007gr indicate the positions of the CI lines. SN 1994I also shows the C I features, although these are not as strong as in SN 2007gr, while the other SNe show a broad dip in this region; these variations are probably attributable to the differences in the respective ejecta velocities of the SNe shown here, with the $\mathrm{C}$ I features being smeared out in those with the highest velocities.

line is expected to be the strongest He I transition (Swartz et al. 1993) and therefore should be highly sensitive to very small quantities of helium (Wheeler et al. 1993; Baron et al. 1996).

\subsection{Optical spectra comparison}

In Figs. 8 and 9, we present early and late-time spectral comparisons of SN 2007gr with the SNe Ic sample: SN 1998bw (Sollerman et al. 2000; Mazzali et al. 2001), SN 2003jd (Valenti et al. 2008b), SN 2002ap (Gal-Yam et al. 2002; Kinugasa et al. 2002; Foley et al. 2003), SN 2004aw (Taubenberger et al. 2006) and SN 1994I (Filippenko et al. 1995b; Sauer et al. 2006). All of the spectra have been ordered according to their explosion energies (top spectra resulting from the most energetic) and corrected for reddening and redshift using the values listed in Table 2. We have previously emphasised the remarkable similarity in light curve morphology and evolution between SNe 2007gr and 2002ap. We will see that this similarity does not extend to the spectral line profiles and ejecta expansion velocities.

The near-maximum spectrum of SN $2007 \mathrm{gr}$ resembles that of SN 1994I on day +1 in terms of the presence of all major features. The main differences between these events at this epoch appears to be the deeper absorption of O I $7774 \AA$ relative to Na I $5896 \AA$ and the narrower spectral widths in SN $2007 \mathrm{gr}$. In contrast, the spectra of SNe 1998bw and 2002ap exhibit very broad features as a result of line blending from high-velocity ejected material. The narrow lines of SNe $2007 \mathrm{gr}$ and 1994I allow for a more secure line-identification and a better separation of spectral features. This is especially true for the C I absorptions $(8335,9094,9406 \AA$ ) which are evident in SN 2007gr at $+20.6 \mathrm{~d}$ and are also detected in SN 1994I, although at weaker intensities. For SNe 2003jd and 2004aw, it is possible that the individual $\mathrm{C}$ I lines may be disguised as a single absorption blend at $\sim 9000 \AA$.

SNe 2007gr and 1994I also have better developed P Cygni profiles for the $\mathrm{NaI} \mathrm{D}$ and $\mathrm{Ca}$ II near-IR triplet lines at only two weeks past maximum as a consequence of their rapid evolution. The comparatively slowly evolving SNe 1998bw, 2004aw, and $2003 \mathrm{jd}$, show no sign of these features until $\sim 2$ months.

Four months after B maximum, the spectra of SN 1994I are the most evolved and show only forbidden line emissions. The absence of the photospheric O I $7774 \AA$ emission line also indicates that the evolution from the photospheric to nebular phase is faster than in SNe 1998bw, 2002ap and 2007gr. Even at this nebular phase, the spectral lines of SN 2007 gr remain narrower than the other type Ic SNe presented, indicating the slower expansion velocities of the ejecta. The nebular phase spectra all show [O I] $\lambda \lambda 6300,6364$ as one of their strongest features, complemented with lines of [Ca II] $\lambda \lambda$ 7291, 7323 / [O II] $\lambda \lambda 7300$, 7330, and the near-IR Ca II triplet. The largely isolated nature of the [O I] doublet is found to be narrower in SN 2007gr compared to all the SNe Ic presented including SN $1994 \mathrm{I}$ by $\sim 1000 \mathrm{~km} \mathrm{~s}^{-1}$.

The nebular spectra of SN 2002ap, 1998bw and 2007gr have strong [OI] $\lambda \lambda$ 6300, 6364 and $\mathrm{MgI}] \lambda 4571$ emissions. The ratio of $\mathrm{MgI}$ ] to [O I] may provide some information on the degree of stripping of the outermost layers experienced by the progenitor star, with a higher $\mathrm{Mg} \mathrm{I}] /[\mathrm{OI}]$ ratio implying a greater degree of stripping as more of the $\mathrm{O}-\mathrm{Ne}-\mathrm{Mg}$ layer is exposed (Foley et al. 2003). Additionally, a higher $\mathrm{Mg} \mathrm{I}] /[\mathrm{OI}]$ ratio at later epochs may simply imply that less of the outer $\mathrm{C} / \mathrm{O}$ layer is observed due to the transparency of the ejecta. We note that the $[\mathrm{OI}]$ and $\mathrm{Mg} \mathrm{I}]$ lines may well be formed via different mechanisms, with [O I] being collisionally excited while $\mathrm{Mg} \mathrm{I}]$ arising predominantly due to recombination as suggested by Kozma \& Fransson (1998). This could influence the line ratios that we 


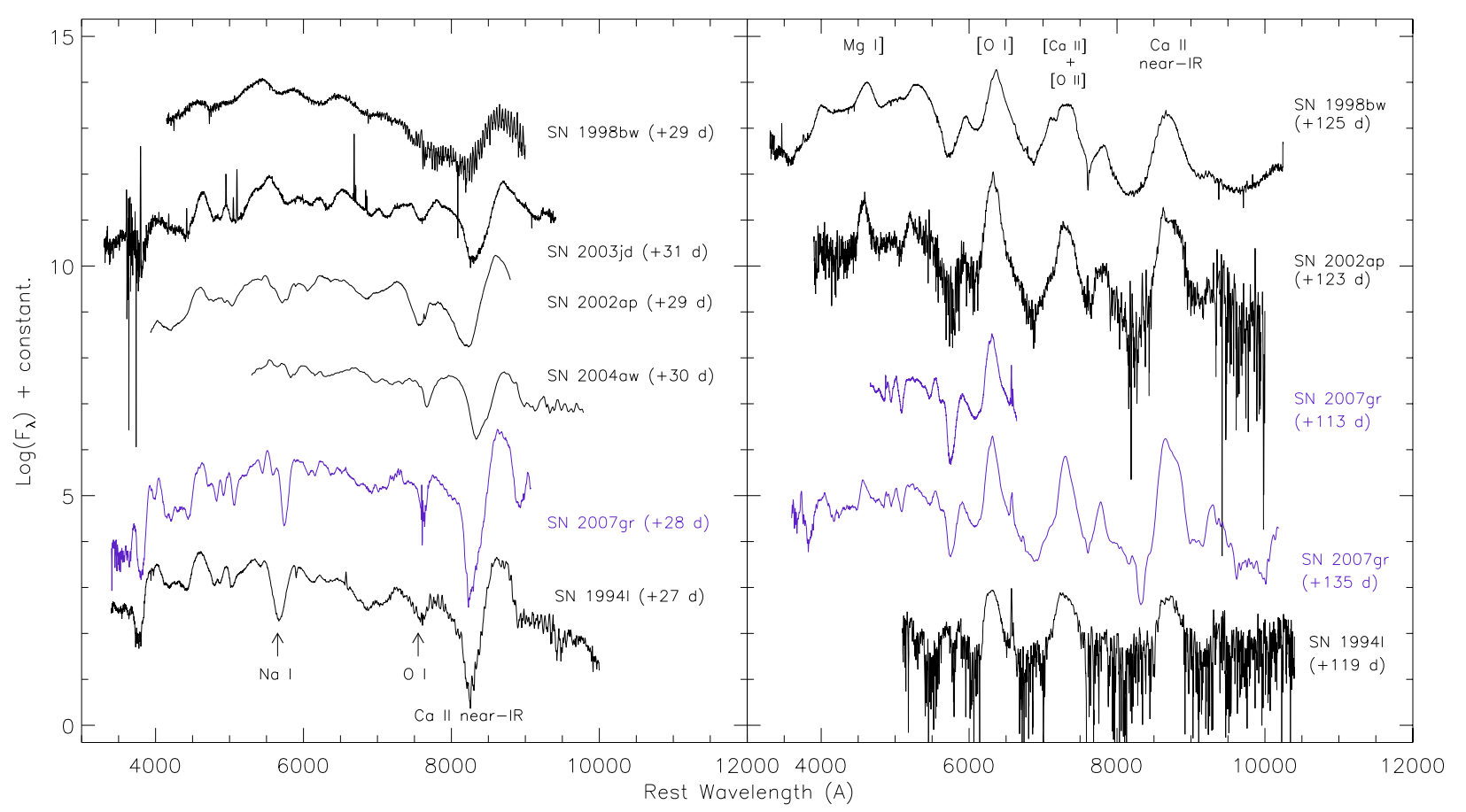

Fig. 9. Comparison of spectra of SNe about one month (left) and four months (right) after $B$ maximum light. The P-Cygni absorptions of Na I D ( $\lambda \lambda$ 5891, 5897), O I ( $\lambda$ 7774) and the Ca II near-IR triplet are clearly marked in the left panel. Typical nebular emission lines are indicated in the right panel.

measure in different SNe. Detailed modelling of the excitation mechanisms for these lines is beyond the scope of this work, so we merely flag this caveat, and use the line ratios as a proxy for the composition.

In Fig. 10 we show the evolution of the $\mathrm{MgI}] /[\mathrm{OI}]$ ratio for a sample of CCSNe during the nebular period +100 to $+400 \mathrm{~d}$. The $\mathrm{MgI}] /[\mathrm{OI}]$ ratio was calculated by first fitting a cubic spline to a local continuum. The continuum was then subtracted so that the integrated flux in the emission lines could be estimated. All of the SNe in Fig. 10 that have more than one epoch of measurement available, show a trend of increasing $\mathrm{MgI}] /[\mathrm{OI}]$ with time, with the marked exceptions of SNe 1998bw and 2006aj. Interestingly, these two SNe are the only $\mathrm{SNe}$ in this sample that were associated with long-duration GRBs. For the SNe for which only a single measurement was possible (SNe 1985F, 1997ef, 2000ew, 2003jd), the Mg I]/[O I] ratio was found to lie within the bounds spanned by other $\mathrm{SNe}$ of this type, and are included in Fig. 10 for completeness.

The increasing $\mathrm{MgI}] /[\mathrm{OI}]$ trend observed for the majority of the SNe sample may simply be due to an abundance effect, as hypothesised by Foley et al. (2003). In symmetric or quasisymmetric explosions, the onion-structure of the progenitor star is almost preserved. This is also true in non-symmetric explosions viewed equatorially. In these cases as time goes by we observe deeper and deeper into the ejecta reaching the $\mathrm{O}-\mathrm{Ne}-\mathrm{Mg}$ shell, and so the $\mathrm{MgI}] /[\mathrm{OI}]$ is expected to increase.

At the beginning of the nebular phase $(+120$ to $+170 \mathrm{~d})$, SN 2007gr has lower Mg I]/[OI] ratios than the other SNe Ic. The lower Mg I]/[O I] ratio of SN 2007gr at these early epochs may suggest that we are still observing the highly concentrated C/O layer of the outer ejecta. This may support the idea that $\mathrm{SN} 2007 \mathrm{gr}$ originated from a carbon-rich progenitor (see Sect. 5.3). Another possible reason for the lower $\mathrm{Mg} \mathrm{I}] /[\mathrm{O} \mathrm{I}] \mathrm{ra}-$ tio may be due to the inefficient burning of the large quantities of carbon in SN $2007 \mathrm{gr}$, thus producing only small quantities of
$\mathrm{Mg}$. Conversely, the larger $\mathrm{Mg}$ I] to [O I] ratios of SN 2002ap may imply that the progenitor experienced greater stripping of the outer carbon layer, since at an epoch similar to SN $2007 \mathrm{gr}$ we are already probing deeper into the core of the progenitor, revealing the O-Ne-Mg layer (e.g. Foley et al. 2003).

The data also show that SNe 2007gr and 2002ap exhibit larger $\mathrm{MgI}$ ] to [O I] ratios than the type Ib $\mathrm{SNe} 1985 \mathrm{~F}$ (Filippenko \& Sargent 1986) and 2007Y (Stritzinger et al. 2009). In this figure, we have also included the ratios of the type IIb SNe 2001ig (Silverman et al. 2009) and 1993J (Fransson et al. 2005). The Mg I]/[O I] ratios of the type IIb SNe are also consistently lower than those of the type Ic SNe 2007gr, 2002ap and 2004aw at the same epochs. The general trend from our sample supports the idea that the progenitors of type Ic SNe experience greater envelope stripping than those of type Ib and IIb.

The decreasing Mg I]/[O I] trend observed for SNe 1998bw and 2006aj may be explained by the rapid rotation of the GRBprogenitors which result in greater mixing (via meridional circulation), and probably induce some degree of asymmetry. A complete mixing of the onion structure is expected in non-symmetric explosions viewed along the polar axis (along the jet). However, it may be that because of the efficiency of this mixing mechanism, the early $\mathrm{MgI}] /[\mathrm{OI}]$ ratios will be close to the average values observed in other Type Ic $\mathrm{SNe}$ (e.g. first point of SN 1998bw). As time progresses, the quantity of $\mathrm{Mg}$ will diminish and since $\mathrm{O}$ is more abundant, its lines will dominate over $\mathrm{Mg}$ lines. In this simple view, the $\mathrm{SNe}$ which show the trend of SNe 1998bw and 2006aj could be jet-like explosions or explosions where the ejecta has been fully mixed. An alternative interpretation may be that the ionisation equilibrium evolves with time for $\mathrm{Mg}$ and $\mathrm{O}$ (which have very different ionisation potentials) simply because of decreasing temperature (cf. Meyerott 1980). This may well be a contributing factor that might account for the observed differences. 


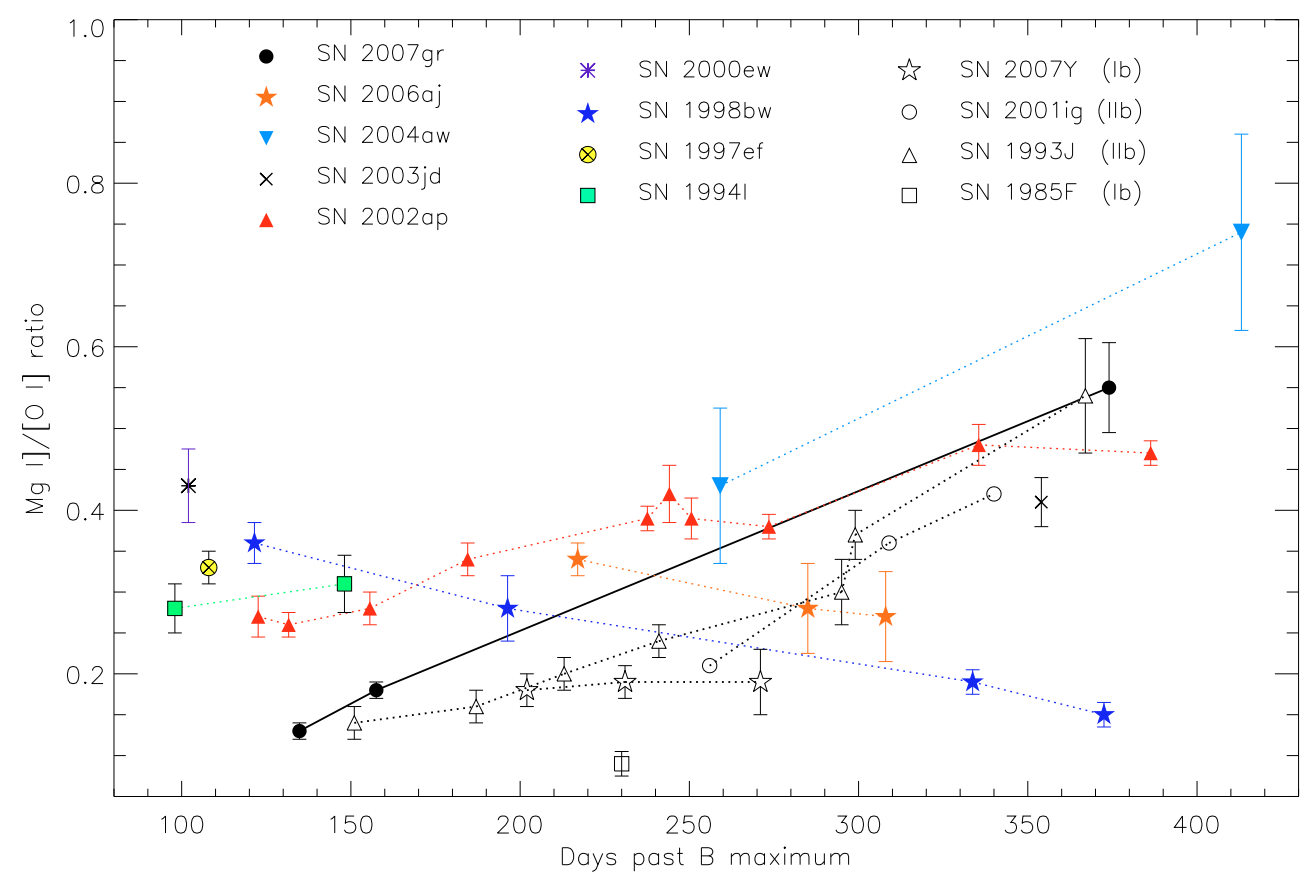

Fig. 10. The $\mathrm{MgI}] /[\mathrm{OI}]$ ratio as a function of time for a sample of SNe. All measurements were performed as described in Sect. 5.2, with the exception of type IIb SN 2001ig, where the measurements were taken from Silverman et al. (2009). All other Ic SNe spectra are from references listed in Table 2 with the exception of SN 2006aj (Mazzali et al. 2007; Taubenberger et al. 2009) and SN 2000ew (Valenti et al., in prep.). The spectra of the type Ib SNe 2007Y (Stritzinger et al. 2009) and 1985F (Filippenko \& Sargent 1986), and the type IIb SN 1993J (Fransson et al. 2005) have also been used. They were corrected for extinction before the measurements were made.

Recent studies of nebular spectra suggest that around half of all $\mathrm{SNe} \mathrm{Ib} / \mathrm{c}$ are strongly aspherical while the remainder appear to have moderate signatures of asphericity (Maeda et al. 2008; Modjaz et al. 2008; Taubenberger et al. 2009; Milisavljevic et al. 2009). As previously mentioned, [OI] $\lambda \lambda 6300,6364$ and $\mathrm{Mg}$ I] $\lambda 4571$ are two of the most dominant features in the latetime spectra of stripped-envelope SNe. As a consequence of their relatively isolated nature and higher abundances to other nebular lines, an analysis of the emission profiles permits one to study the ejecta geometry (Mazzali et al. 2005). In particular, one might expect $\mathrm{Mg}$ and $\mathrm{O}$ to have a similar spatial distribution within the ejecta (e.g. Maeda et al. 2006) and thus the profiles of these emission lines should also be similar.

Unfortunately, a direct comparison of the $\mathrm{Mg} \mathrm{I}]$ and [O I] lines is not possible since the [O I] doublet arises from two forbidden transitions which share the same upper level $\left({ }^{3} \mathrm{P}_{1,2}-{ }^{1} \mathrm{D}_{2}\right)$. The relative intensity of the $\lambda 6300$ and $\lambda 6364$ lines will depend on the OI density and may vary from 1:1 to the low-density limit of 3:1 (Spyromilio 1991). Taubenberger et al. (2009) suggested that the density in stripped envelope $\mathrm{SNe}$ should, after $100 \mathrm{~d}$, have decreased enough to fix the line intensity ratio to 3:1, but recently Milisavljevic et al. (2009) suggested a ratio $1: 1$ to explain the OI $\lambda \lambda 6300,6364$ profiles in 4 stripped envelope SNe. In order to investigate which line intensity ratio is more appropriate for SN $2007 \mathrm{gr}$, we have displayed the profiles of [OI] $\lambda \lambda$ 6300, 6364 and $\mathrm{MgI}$ I] $\lambda 4571$ in Fig. 11 (left panel) at $+158 \mathrm{~d}$ after maximum. Since the profiles of $\mathrm{MgI}]$ $(+$ an artificial second component with a line intensity ratio of 0.33 and the same velocity offset as found in the oxygen doublet) and [OI] are similar, this tells us that the ratio between O I 6300 and 6364 is close to 3:1. We also performed a comparison between [O I] $\lambda \lambda 6300,6364$ and [O I] $\lambda 5577$ ( + an artificial second component with ratio $0.33,0.66,1.00$ ) without obtaining a good match. The [OI] emission profile contains one peak positioned at a blueshift velocity of $\sim-2000 \mathrm{~km} \mathrm{~s}^{-1}$ and the other skewed at $\sim-800 \mathrm{~km} \mathrm{~s}^{-1}$. Taubenberger et al. (2009) found an average blueshift of $\sim 1000 \mathrm{~km} \mathrm{~s}^{-1}$ for the [O I] $\lambda 6300$ line centroids of a large sample of Type $\mathrm{Ib} / \mathrm{c} \mathrm{SNe}$ which is also consistent with SN 2007gr. The authors suggest that the observed effect is probably related to the ejecta geometry and may be a signature of a one-sided explosion, or, more likely, residual opacity in the inner ejecta.

We also present the evolution of the permitted and forbidden lines of $\mathrm{O}$ and $\mathrm{Mg}$ at phases $>+100 \mathrm{~d}$ (centre and right panels of Fig. 11). The figures show that the permitted lines of OI $\lambda 7774$ and Mg I $\lambda 15025$ behave very differently than the forbidden lines of [OI] $\lambda 5577$ and $\mathrm{MgI}] \lambda 4571$. The emission peaks of the permitted profiles are found to be almost centered at their rest wavelengths while those of the forbidden lines are strongly blueshifted, with the effect becoming progressively weaker with time. In addition, the [O I] profile at $5577 \AA$ appears to be even more strongly blue-shifted compared with other forbidden lines.

The observed trend of blueshifted forbidden line centroids compared to the central permitted lines suggest that they appear in different regions of the ejecta since we know that the forbidden lines emerge from a lower-density medium. The systematic effective blue-shift of the forbidden lines may well be due to the residual opacity of the intervening material such that the photons emitted on the rear side of the SN are absorbed along the line of sight.

Taubenberger et al. (2009) and Milisavljevic et al. (2009) provide alternative explanations for the observed trend, including dust formation and contamination from other ions. However, in Sect. 5.5 we claim that dust has not yet formed in the ejecta of SN $2007 \mathrm{gr}$. Additionally, any dust that is present should actually result in a line blueshift which increases with time, not the contrary. Contamination due to other emission lines may also be 


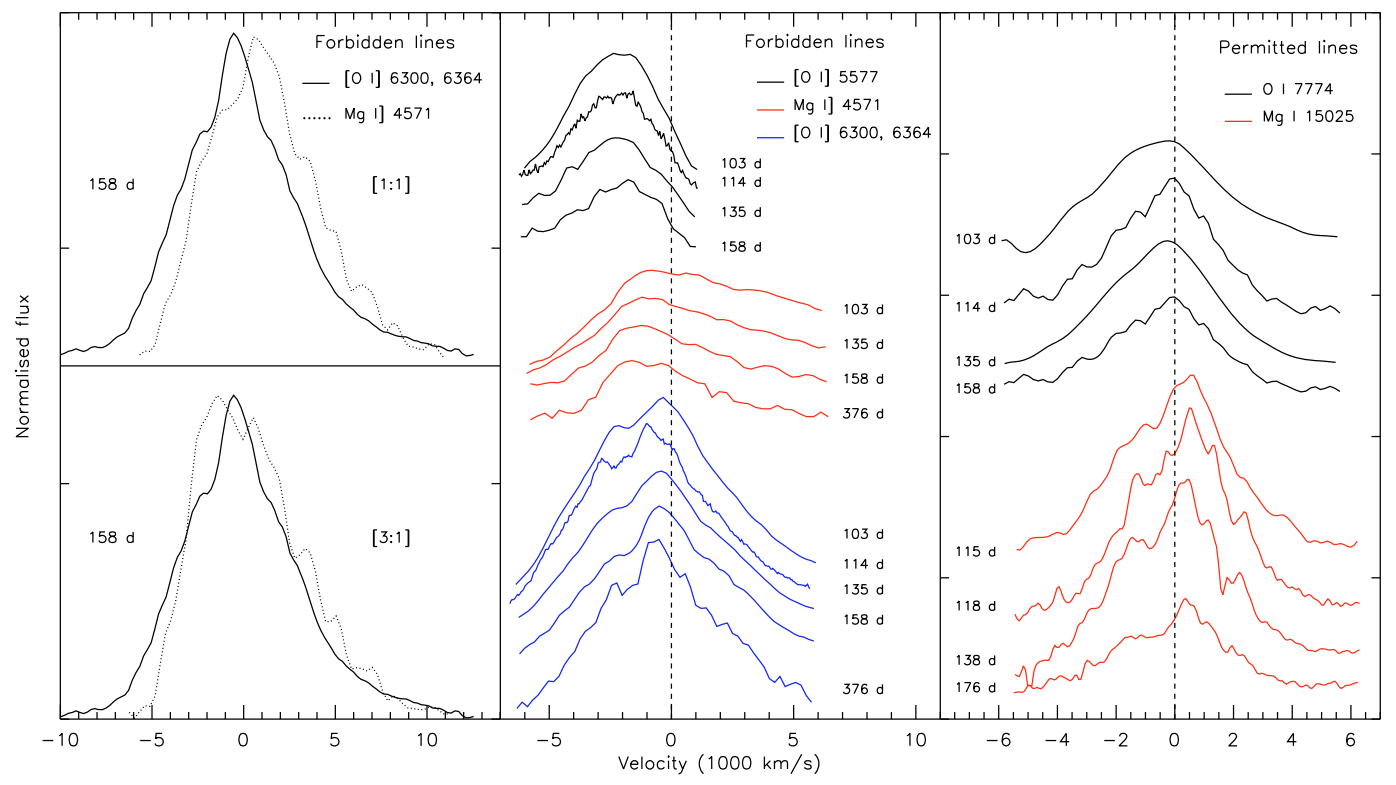

Fig. 11. Left: the profiles of [OI] 6300,6364 and Mg I] 4571 at $+158 \mathrm{~d}$ past $B$ maximum. The profile of [OI] is plotted in velocity space using a rest wavelength of $6315 \AA$, as obtained via Gaussian fitting from the modified profile of [O I] in the normal and low-density limits, 1:1, 3:1. The $\mathrm{Mg}$ I] profile includes the contribution of an artificial component with an intensity ratio of 1 and 0.33 (top and bottom panel, respectively) relative to the real peak and the same velocity offset as found in the oxygen doublet. The peaks of the MgI] profiles have been scaled to match the peak of the oxygen doublet at $+158 \mathrm{~d}$. Centre: evolution of forbidden [OI] 5577, Mg I] 4571 and [O I] 6300, 6364. Right: evolution of the profiles of the permitted lines of O I $7774 \AA$ and Mg I $15025 \AA$. The vertical line in the panels correspond to zero velocity i.e. the rest wavelengths of the line profiles.

neglected due to the similarity of the $[\mathrm{OI}]$ and $\mathrm{MgI}]$ profiles, while contamination on the [O I] $\lambda 5577$ cannot be excluded.

For SN 2007gr, we note that Tanaka et al. (2008) report non-zero polarization across the Ca near-IR triplet at $\sim+21 \mathrm{~d}$ post-maximum. They concluded that their observations could be interpreted either in the context of a bipolar explosion with an oblate photosphere viewed from a mildly off-axis line-of-sight, or a spherically symmetric photosphere with a clumpy Ca II distribution. Out to about $+400 \mathrm{~d}$, the optical and near-IR spectra, show no evidence of double-peaked profiles as would be expected for a significantly aspherical explosion. From our data, we conclude that the explosion of SN 2007gr could not have been more than mildly aspherical.

\subsection{Near-infrared spectra}

All near-infrared spectra of SN 2007gr are presented in Fig. 12. At early epochs, the shape of the NIR continuum is nicely approximated by a black body continuum. The earliest spectra are almost featureless with few lines due to intermediate mass elements such as Mg I, O I, Na I. After maximum light, lines due to the iron group elements begin to appear.

One of the most prominent features in the near-IR spectra is an absorption at $\sim 1.04 \mu \mathrm{m}$. The origin of this feature has been discussed widely by several authors, especially in the context of type Ia SNe, with Wheeler et al. (1998) favouring Mg II and Meikle et al. (1996) suggesting a blend of Mg II and He I $1.083 \mu \mathrm{m}$; the latter is a line that is easily excited in a variety of astrophysical contexts. In fact, other elements such as C, $\mathrm{O}, \mathrm{Si}$, and $\mathrm{Fe}$ may also contribute to features in this region.

If He I were indeed responsible for the absorption, it would only be detected if the lower level of $\mathrm{He}$ becomes highly populated in the presence of high energy, non-thermal radiation. The source of this radiation would be the $\gamma$-rays produced in the radioactive decay of ${ }^{56} \mathrm{Ni}$ to ${ }^{56} \mathrm{Co}$. The lower level population may then absorb photospheric photons of $\sim 1$ micron yielding the P Cygni profile. Mazzali \& Lucy (1998) were able to reproduce the 1.05 micron feature in the type Ia SN 1994D using just $0.01 M_{\odot}$ of helium, implying that only small quantities are needed to account for this feature. Thus the apparent lack of strong He lines in type Ic SN spectra may imply a low abundance of $\mathrm{He} \mathrm{I}$ and/or insufficient mixing of ${ }^{56} \mathrm{Ni}$ into the outermost layers of the ejecta.

If $\mathrm{HeI}$ were a major contributor to the $1.04 \mu \mathrm{m}$ feature in SN 2007gr, this would imply an average expansion velocity of $\sim 13000 \mathrm{~km} \mathrm{~s}^{-1}$ at $+15.5 \mathrm{~d}$ which later declines to $\sim 10000 \mathrm{~km} \mathrm{~s}^{-1}$ by $+114.5 \mathrm{~d}$. In spite of the fact that SN $2007 \mathrm{gr}$ is a narrow-lined Ic, so that the identification of lines should be facilitated, the optical region is plagued by blends, especially in the regions where $\mathrm{HeI}$ lines would lie e.g. the He I $3888 \AA$ line lies close to the Ca II H \& K lines and the He I 5875 A lies close to the NaI D. The possible presence of the He I line at $5875 \AA$ is suggested in Sect. 5.4 to account for the unusual velocity distribution of the Na I D feature. In the near-IR, one might expect to detect He I $2.0581 \mu \mathrm{m}$. However, we see no evidence of any feature at this wavelength in our entire near-IR spectral series.

Although He I $2.0581 \mu \mathrm{m}$ is not visible in SN $2007 \mathrm{gr}$, we cannot fully disregard the contribution of helium to the $1.04 \mu \mathrm{m}$ absorption. The longer decay time from the $2 \mathrm{~s} 3 \mathrm{~S}$ level of $\mathrm{He}$ I $1.0830 \mu \mathrm{m}$ means that this line can behave much like a lowenergy, ground state transition, unlike the faster $\left(\times 4.5 \times 10^{5} \mathrm{~s}\right)$ decay time of He I $2.0581 \mu \mathrm{m}$, and so will be easier to excite. Thus the absence of the He I $2.0581 \mu \mathrm{m}$ line does not necessarily mean that the $1.0830 \mu \mathrm{m}$ line does not contribute to the $1.04 \mu \mathrm{m}$ feature. This cannot be quantified without detailed modelling which is beyond the scope of this paper. Yet, we can conclude that if $\mathrm{He}$ is present in SN $2007 \mathrm{gr}$, the amount must be small.

Valenti et al. (2008a) suggested that the $1.04 \mu \mathrm{m}$ feature in SN 2007 gr may be attributed primarily to the C I ion (rest wavelength at $1.0695 \mu \mathrm{m}$ ). They found that earlier (optical) spectra 


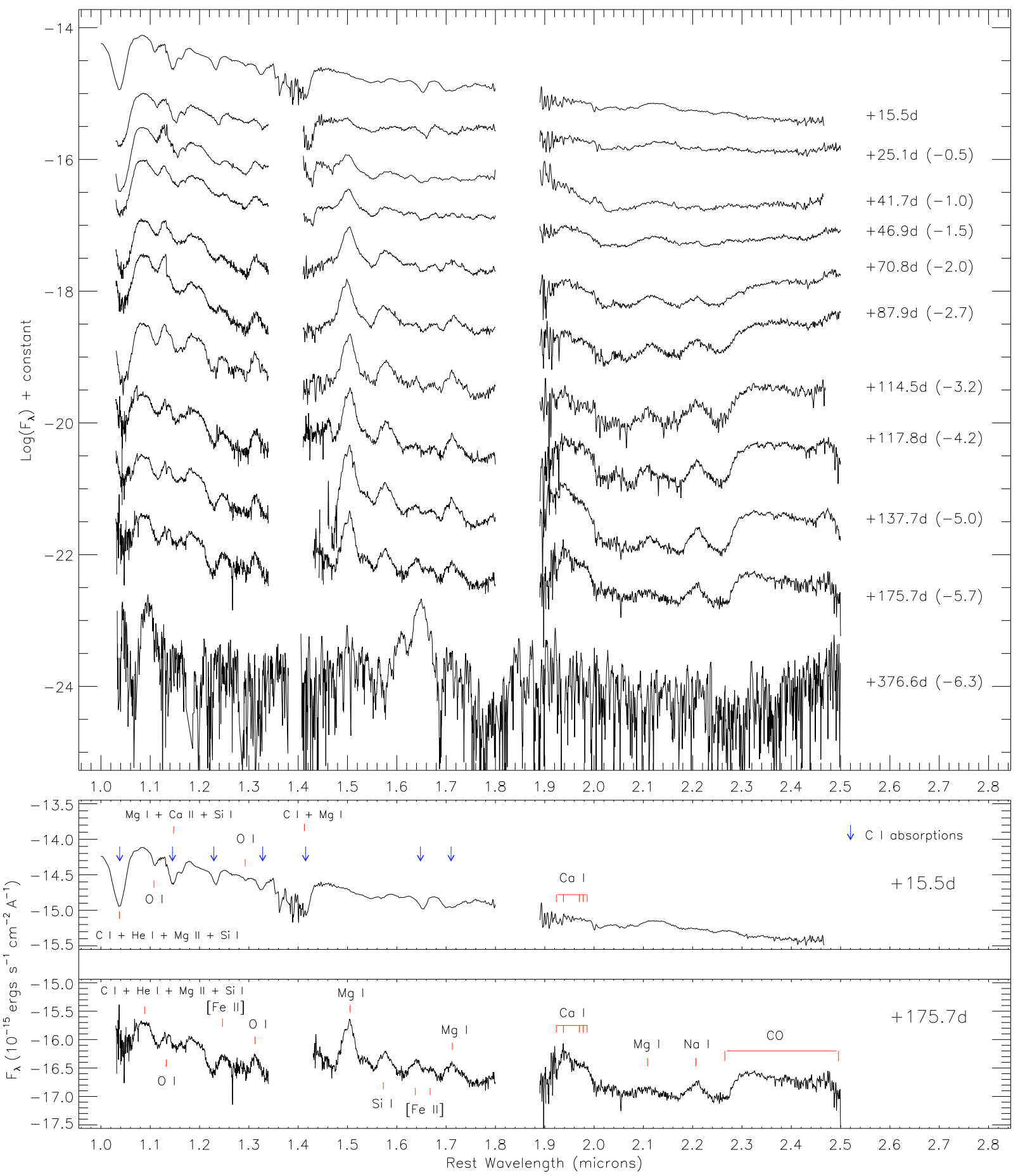

Fig. 12. The near-infrared spectral evolution of SN 2007gr. The spectra are displayed in the rest frame of NGC 1058. To increase the temporal coverage we include the spectrum obtained by Valenti et al. (2008a) on day +15 . The $+376.6 \mathrm{~d}$ spectrum has been smoothed with a box size of 5 pixels. The spectra have been displaced vertically for clarity by the numbers shown in brackets. The rest wavelengths of possibly identified lines are also included.

showed evidence for C II features at very early epochs $(\sim-8 \mathrm{~d})$ which fade rapidly, to be replaced by lines due to C I. From an initial modelling effort, they obtained a fairly reasonable match to their optical-near-IR spectrum at day +15 , also reproducing several other observed CI lines. However, even with a model enhanced in $\mathrm{CI}$, the entire $1.04 \mu \mathrm{m}$ feature, and the blue wing in particular, could not be matched. They suggested that this could be due to the contribution of one or more other ions e.g. Mg II, Si II. Indeed Millard et al. (1999) also had difficulty in accounting for this infrared absorption feature in SN 1994I with
He I alone, suggesting that it could be a blend of He I and C I lines.

Together with the $1.04 \mu \mathrm{m}$ feature, the nebular spectra of the $J$ band reveal emission lines of Fe II, Si II, Mg I, Na I, O I and $\mathrm{S}$ I, while the $H$ band is dominated by emission features produced by iron-group elements (Fe II). The $K$ band spectra appear to contain emission features from $\mathrm{Na}$ I, $\mathrm{Mg}$ II and the first overtone of CO (see also Fig. 16). We will discuss this in Sect. 5.5. The [Fe II] lines at $\lambda_{\text {rest }}=1.257 \mu \mathrm{m}$ and $\lambda_{\text {rest }}=1.644 \mu \mathrm{m}$ are particularly prominent in the $J$ and $H$ bands, respectively. These 


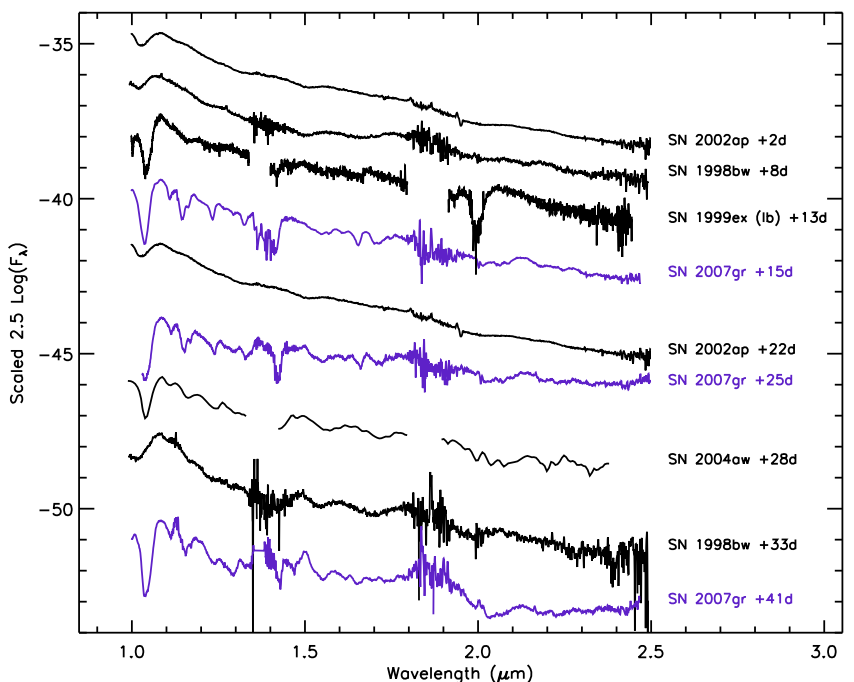

Fig. 13. Comparison of the near-IR spectra of SN 2007gr to $\mathrm{SNe}$ 2002ap (broad-lined Ic), 1998bw (broad-lined Ic), 1999ex (type Ib), and 2004aw (type Ic). Note the presence of the prominent He I 1.083, and $2.0581 \mu \mathrm{m}$ lines in the spectrum of SN 1999ex. See text for references.

are complemented with unblended Fe II lines in the optical at expansion velocities $\sim 4000-6000 \mathrm{~km} \mathrm{~s}^{-1}$.

The broad feature positioned at $\sim 1.13 \mu \mathrm{m}$ can be identified with the $1.1287 \mu \mathrm{m}\left(3 \mathrm{p}^{3} \mathrm{P}-3 \mathrm{~d}^{3} \mathrm{D}^{0}\right)$ O I transition (Meikle et al. 1989). The $\operatorname{MgI}(1.503 \mu \mathrm{m})$ feature observed in the $H$ band is matched by strong features of Mg II at $4481 \AA$ in the optical region. The $K$ band region of the NIR contains the $2.207 \mu \mathrm{m}$ doublet of $\mathrm{Na}$ I. In the optical too, $\mathrm{Na}$ I dominates the region around $5900 \AA$ from the early to very late phases. Features which appear near 1.94 and $1.98 \mu \mathrm{m}$ around $+138 \mathrm{~d}$ can probably be identified with Ca I.

Currently, few near-IR spectra of Ib/c SNe are found in the literature. This is particularly true at late phases. At earlier epochs, the situation is somewhat less dire and we compare our early time spectra of SN 2007gr with those of SNe Ic 2004aw (Taubenberger et al. 2006), 1998bw (Patat et al. 2001), the SN Ib 1999ex (Hamuy et al. 2002), and previously unpublished spectra of SN 2002ap (Ic) ${ }^{5}$. The near-IR spectra of all SNe mentioned above are presented in Fig. 13.

The broad-lined spectra of SNe 2002ap and 1998bw appear almost featureless, with the exception of a weak absorption at $\sim 1.04 \mu \mathrm{m}$. This behaviour probably reflects the severe line blending due to high ejecta velocities in these objects. In contrast, the distinct narrow features of SN $2007 \mathrm{gr}$ are clearly visible at similar epochs. The type Ib SN 1999ex exhibits equally strong He I absorption features at both $1.04 \mu \mathrm{m}$ and $\sim 2.0 \mu \mathrm{m}$. This is supported by the presence of He I lines in the optical spectra (Hamuy et al. 2002). Interestingly, all SNe shown in Fig. 13 display a prominent feature near $1.04 \mu \mathrm{m}$ regardless of epoch, however with the exception of SN 1999ex none shows an absorption near $2.0 \mu \mathrm{m}$ at any time. The $1.04 \mu \mathrm{m}$ absorption feature in SN 2007gr appears to be stronger than in SN 1999ex, providing some support for the contribution of C I.

\footnotetext{
5 These spectra were obtained at the UKIRT telescope and reduced using standard procedures.
}

\subsection{Ejecta velocity}

The velocities of several ejecta lines were determined by fitting a Gaussian profile to the absorption component of the P-Cygni feature in order to measure the position of the blue-shifted minimum. This method provides an estimate of the expansion velocities of the layers where the individual lines predominantly form and therefore the distribution of elements within the ejecta. Only the velocities of a few spectral features have been measured since for many others line blending is a problem, even for the relatively low velocities of SN $2007 \mathrm{gr}$. The evolution of ejecta velocities is shown in Fig. 14.

The CaII velocities of both the $\mathrm{H} \& \mathrm{~K}$ features as well as the near-IR triplet are significantly higher than those of $\mathrm{Mg}$ II (4481 $)$, Fe II (4924, 5018, 5170 $)$, Na I (5890-5896 ), O I (7774 $\AA$ ), C I (9087, 10695 and $11753 \AA$ ). Assuming homologous expansion, the higher velocity Ca II lines imply that they form further out in the ejecta than the O I, Na I, and Fe II lines which emerge from the inner ejecta layers. The formation of $\mathrm{Ca}$ II lines further out is predominantly because these lines are extremely strong and therefore become optically thick very rapidly, even in the low density, outer layers of the ejecta. As expected, the absorption component of the PCygni minima gradually shift redwards with time revealing the deeper and more slow moving ejecta.

Comparisons of velocity widths of the $\mathrm{NaI} \lambda$ 5891, Si II $\lambda$ 6355, O I $\lambda 7774$ and the Ca II NIR triplet for SNe 2007gr, 2004aw, 2002ap and 1994I are shown in Fig. 15. The expansion velocity $v_{\text {exp }}$ of SNe Ic can be directly measured from the minima of spectral absorption lines, of which the Si II line $(6355 \AA)$ is commonly used when present. Assuming that the blue minimum contains no contamination from other ion species, the derived $v_{\text {exp }}$ values of SN $2007 \mathrm{gr}$ reveal comparatively low velocities which are probably related to the narrow features observed in the spectra. Using the Si II velocity as an approximation, we estimate a photospheric velocity of $\sim 6700 \mathrm{~km} \mathrm{~s}^{-1}$ at $B$ maximum, which decreases to only $\sim 4000 \mathrm{~km} \mathrm{~s}^{-1}$ ten days later.

In contrast, the photospheric velocity of SN 2002ap declines rapidly and ranges from $\sim 17000$ to $7000 \mathrm{~km} \mathrm{~s}^{-1}$ in a similar time frame. The blue-shifts of the Si II line of these two objects are comparable at two weeks from $B$ maximum, however the spectral features of SN 2002ap remain broader than those of SN 2007gr. This suggests that the differences between SNe 2007gr and 2002ap result from the differences in their density profiles, i.e. a steeper density gradient for SN $2007 \mathrm{gr}$ and hence a smaller velocity range at which the lines can form. The photospheric velocity of SN $2007 \mathrm{gr}$ is also similar to that of SN 1994I about one week after B maximum, implying that the kinetic energy to unit mass should be also similar at this epoch.

An interesting point to note is that the measured expansion velocities for Na I 5890, $5896 \AA$ about two weeks post-maximum show an increase for all Ic SNe in Fig. 15 with the exception of SN 2004aw, followed by a plateau. A possible explanation for this behaviour may be the contribution of another line. A likely possibility is the contribution from He I $\lambda 5876 \AA$ which has been previously discussed by Clocchiatti et al. (1996), although as noted previously other strong lines of He are not obvious in the optical spectra. In addition to the possibility of contamination from $\mathrm{He}$ I, the recombination of $\mathrm{Na}$ II to $\mathrm{NaI}$ may be an important mechanism which contributes to the strength of the Na I line in different velocity ranges. The observed behaviour may also be explained by the departure from LTE to NLTE, again affecting the strength of the Na ID feature. 


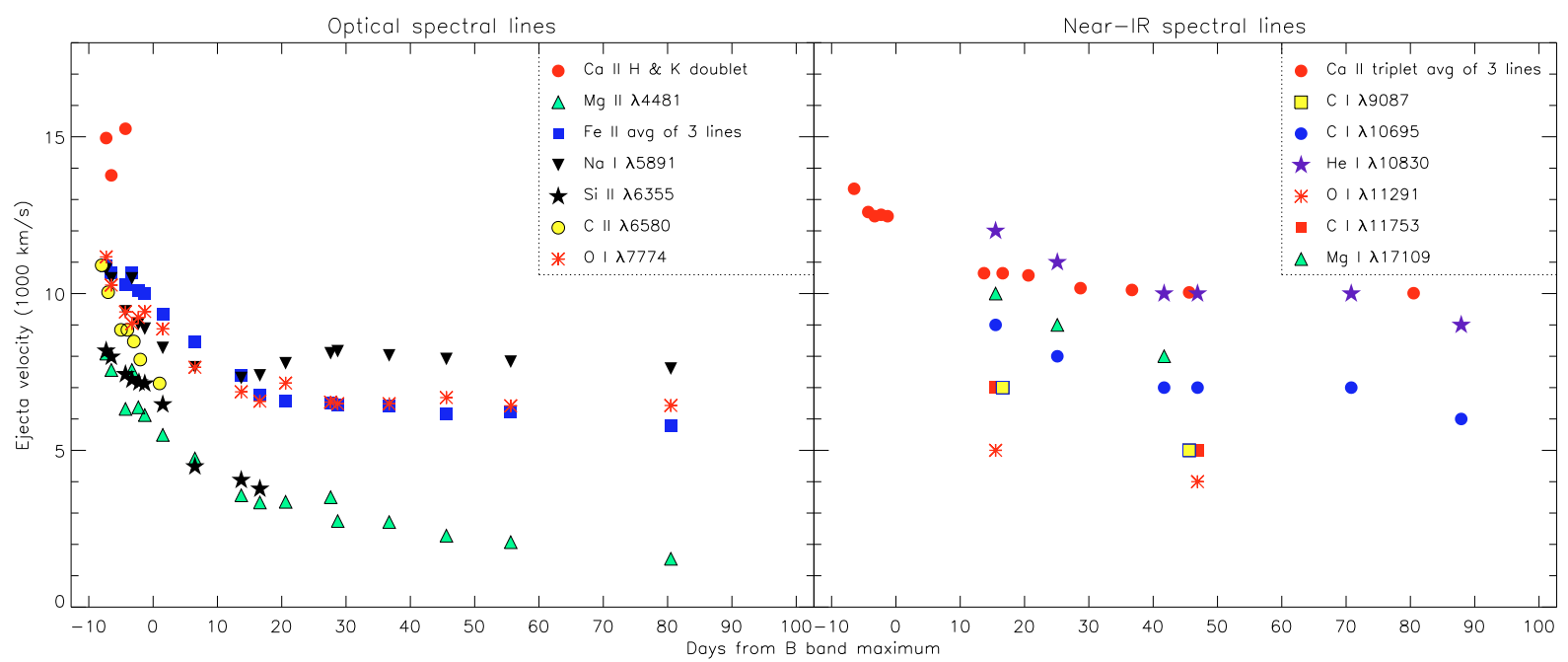

Fig. 14. Evolution of the velocities from the different spectral lines observed in SN 2007gr. The errors in the measured velocities are estimated to be $\sim 10 \%$. The minimum error is not less than $500 \mathrm{~km} \mathrm{~s}^{-1}$.
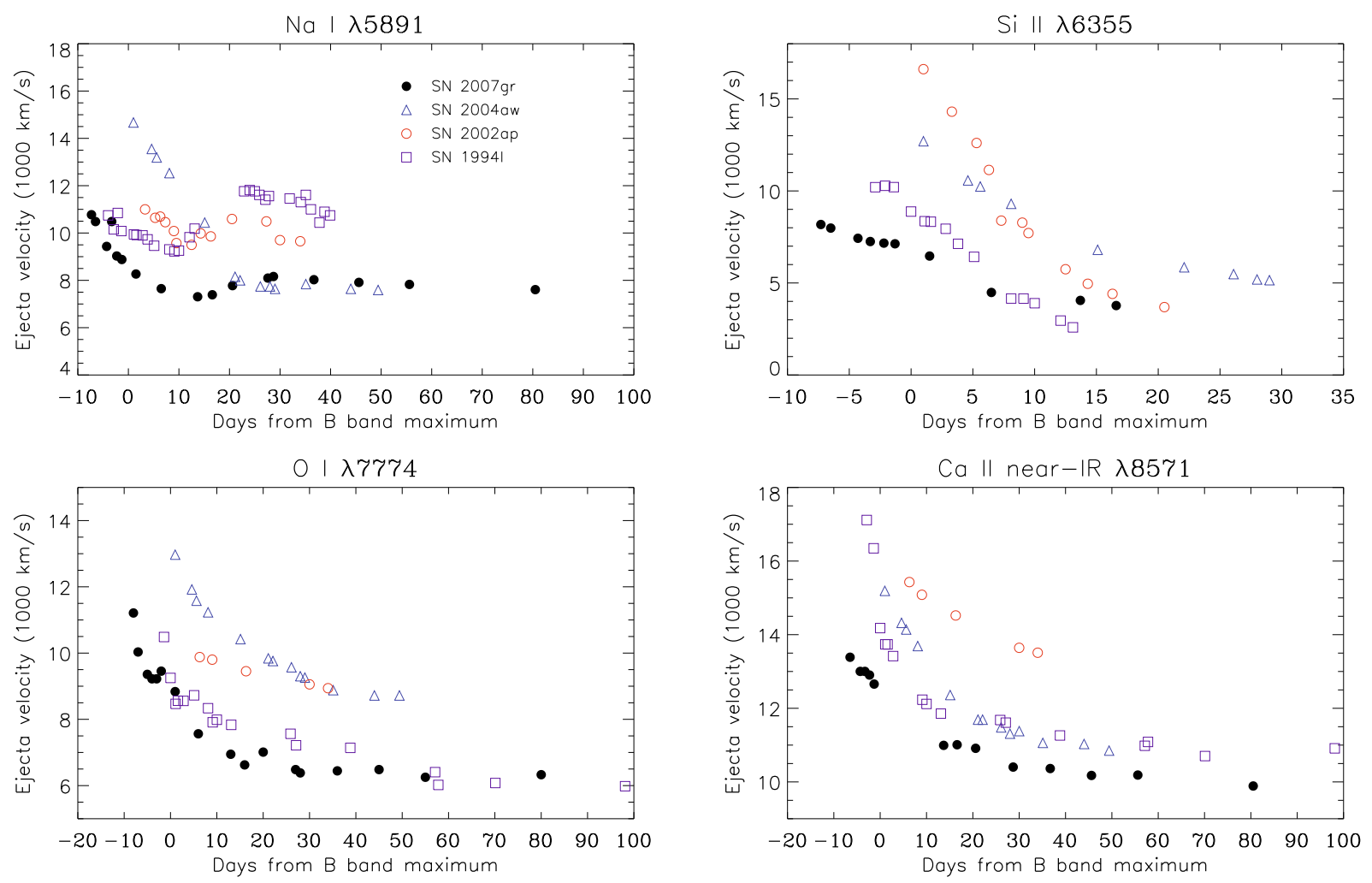

Fig. 15. Comparison of the velocity evolution of the type Ic SNe 2007gr, 2004aw, 2002ap, and 1994I using different spectral lines.

\subsection{Carbon monoxide emission}

Carbon monoxide emission in a supernova was first reported by Catchpole \& Glass (1987) in SN 1987A. Spectroscopic coverage of the first overtone $(\Delta v=2)$ spanning days $\sim 110-574$ was presented by Meikle et al. (1993) while coverage of the fundamental $(\Delta v=1)$ band emission spanning days $\sim 157-615$ was presented by Bouchet \& Danziger (1993) and Wooden et al. (1993). Since then the first overtone of $\mathrm{CO}$ has been observed in numerous type II SNe with detections in the spectra of SN 1995ad (Spyromilio \& Leibundgut 1996), SN 1998S (Gerardy et al. 2000; Fassia et al. 2001) and SNe 1998dl \& 1999em (Spyromilio et al. 2001).
With the sole exception of SN 1987A, observations of the $\mathrm{CO}$ fundamental band (near $\sim 4 . \mu \mathrm{m}$ ) have remained elusive, given the challenging nature of the observations. However, with space-based instrumentation, the $\mathrm{CO}$ fundamental has also been observed in several IIP SNe: SNe 2004dj, 2005af, 2004et (Kotak et al. 2005, 2006, 2009). Although CO emission in type II seems to be a common occurrence, detections in type Ic have been limited to SN 2000ew (Gerardy et al. 2002) and now SN 2007gr.

The subject of molecular emission in SNe has been extensively reviewed by Petuchowski et al. (1989), Lepp et al. (1990), Dalgarno et al. (1998) and Gearhart et al. (1999), with particular reference to SN 1987A. The main formation mechanism is the radiative association of $\mathrm{C}$ and $\mathrm{O}$ atoms whereby photons are emitted and bound $\mathrm{CO}$ molecules are formed. With time, the flux 
of the $\mathrm{CO}$ emission will decrease as a result of destructive interactions with fast electrons which are produced by the $\gamma$-rays from the decay of ${ }^{56} \mathrm{Co}$. Additionally, the presence of ionised $\mathrm{He}$ that is microscopically mixed within the carbon-oxygen layers could destroy the $\mathrm{CO}$ molecules via charge transfer reactions:

$\mathrm{He}^{+}+\mathrm{CO} \rightarrow \mathrm{C}^{+}+\mathrm{O}+\mathrm{He}$.

Thus molecular emission is a useful diagnostic for examining the conditions and degree of mixing in the ejecta of SNe.

We see clear evidence for the presence of the first-overtone of $\mathrm{CO}$ emission from $2.29 \mu \mathrm{m}$ to the red end of the spectrum at $2.4 \mu \mathrm{m}$. This feature first appears at $+70 \mathrm{~d}$ post- $B$ maximum which is the earliest reported detection of $\mathrm{CO}$ in any type of SN. In part, this might be due to the lack of suitable data for the majority of SNe. However, the early appearance of CO is in line with its relatively rapid evolution of SN $2007 \mathrm{gr}$ compared to other SNe for which CO has been detected (e.g. SN 1987A) and is also consistent with the idea that the progenitor of SN $2007 \mathrm{gr}$ was a compact star. In earlier sections we have argued against the presence of large amounts of He in the ejecta of SN 2007gr, so the fact that CO is detected at all would seem to indirectly support this idea. Although one might invoke the possibilities that ionised He was not microscopically mixed with the underlying C/O rich layers (Lepp et al. 1990; Gerardy et al. 2000, and references therein), or that a significant quantity of He was not ionised, both possibilities seem contrived in view of the observation that strong spectral signatures due to $\mathrm{He}$ are not convincingly detected over a time-span of more than a year.

The CO feature grows in strength up to day +138 before fading. In SN 1987A, a similar trend was observed, except that the intensity of the feature increased until $\sim+200 \mathrm{~d}$. This is believed to be due to the non-uniform deposition of energy at early times as a result of the greater optical depth of $\gamma$-rays in the iron core. After sufficient expansion of the ejecta by late times, the optical depth has decreased, and greater energy deposition occurs in layers other than the iron core, i.e. the C/O layer (Liu \& Dalgarno 1995). Thus the energy deposition becomes progressively uniform.

Figure 12 shows that by day +176 , the $\mathrm{CO}$ emission has faded by a factor of $\sim 1.5$ compared to day +138 beyond $2.35 \mu \mathrm{m}$. This decrease in flux may represent the enhanced energy deposition rate into the $\mathrm{C} / \mathrm{O}$ layers from fast electrons.

As mentioned by Gerardy et al. (2002), the shape of the $\mathrm{CO}$ profile provides a lower limit to the temperature of the emitting region and so band profile modeling can be used to infer the excitation temperature. Since observations of $\mathrm{CO}$ emission in type Ic are rather limited, a comparison with the molecular emission in the spectra of SN 1987A is a good alternative. In Fig. 16 we compare our $K$-band spectrum of SN $2007 \mathrm{gr}$ at day +137.7 to those of SN 1987A (Meikle et al. 1989) and the type IIP SN 2004dj (Kotak et al. 2005). The spectra have been scaled so that the initial slopes of the CO emissions near $2.24 \mu \mathrm{m}$ are relatively well matched. In the +137.7 day spectrum of SN $2007 \mathrm{gr}$, the $\mathrm{CO}$ feature decreases in flux at longer wavelengths in a similar manner to both SNe 1987A and 2004dj. However, the first two band heads (labelled at $\sim 2.31$ and $2.33 \mu \mathrm{m}$ ) of SNe 1987A and 2004dj can be clearly observed while that of SN $2007 \mathrm{gr}$ is not as distinct. LTE modelling of CO has shown that the two band heads have comparable strengths at temperatures $<2000 \mathrm{~K}$ (Sharp \& Höflich 1989; Liu et al. 1992). We thus conclude that the temperature of the CO emitting region in SN 2007gr is greater than $2000 \mathrm{~K}$.

An approximate velocity of the $\mathrm{CO}$ emission can be obtained by comparing our spectra of SN $2007 \mathrm{gr}$ with the higher

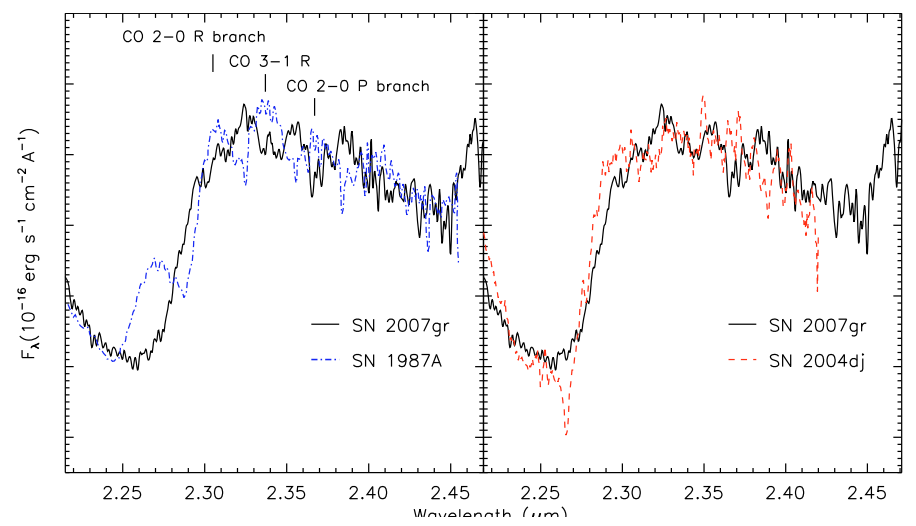

Fig. 16. Left: the CO emission profile of SN $2007 \mathrm{gr}(+137.7 \mathrm{~d}$ post $B$ maximum) overlaid with the $192 \mathrm{~d}$ (post-explosion) spectrum of SN 1987A. The features indicated refer to SN 1987A. Right: CO profile of SN $2007 \mathrm{gr}$ at $+137.7 \mathrm{~d}$ (post- $B$-maximum) overlaid with that of SN 2004dj at $137 \mathrm{~d}$ post-explosion.

resolution spectrum of SN 1987A. The slope of the 2-0 R-branch band head indicates that the expansion velocity in SN $2007 \mathrm{gr}$ is approximately similar to SN $1987 \mathrm{~A}\left(1800-2000 \mathrm{~km} \mathrm{~s}^{-1}\right.$ Spyromilio et al. 1988), which was also the case for SNe 1998dl and 1999em (Spyromilio et al. 2001).

CO was still detected in SN 1987A as late as $574 \mathrm{~d}$ after explosion (Meikle et al. 1993), whereas it had disappeared by day 355 in the type IIn SN 1998S (Gerardy et al. 2000).

For SN 2007gr, we notice a fading of the feature by day +176 , and we do not detect it in the $+377 \mathrm{~d}$ spectrum. However, we cannot entirely rule out the presence of the $\mathrm{CO}$ overtone since the $\mathrm{S} / \mathrm{N}$ is not sufficiently high. As suggested by Liu et al. (1998), the disappearance of molecular emission may be due to the rapid decline in temperature as a result of the efficient cooling by $\mathrm{CO}$ at later times (decrease in heating rate and increasing departure from LTE).

Aside from the diagnostic constraints e.g. on microscopic mixing, that are provided by $\mathrm{CO}$, the presence of molecules in the ejecta provides an additional source of cooling. This may be conducive to the formation of dust in the ejecta. One might expect the combination of molecules and low velocities observed in SN 2007gr to result in some condensation of dust grains, particularly graphite grains for the carbon-rich progenitor of SN 2007gr (Valenti et al. 2008a). However, at the epoch of our last near-IR spectrum ( $\sim 1$ year), we do not see any clear evidence of a rising continuum in the $K$-band. The $H$-band light curve does not show any sign of flattening but appears to follow the ${ }^{56} \mathrm{Co}$ decay rate reasonably well (see Fig. 4). The $H-K$ colour evolution of SN $2007 \mathrm{gr}$ argues against dust formation with a relatively blue $H-K$ colour of $\sim-1.1 \mathrm{mag}$ at $\sim+400 \mathrm{~d}$. Additionally, dust formation should result in a blueshift in the peaks of emission lines which increases with time. We observe no such effect in the evolution of the emission features in the optical or near-IR spectra of SN $2007 \mathrm{gr}$ (see Figs. 7 and 12, respectively).

In relation to other core-collapse $\mathrm{SNe}$ types, IR signatures of dust condensation were found in the ejecta of the type IIP SN 1987A as early as $100 \mathrm{~d}$ post-explosion (Suntzeff \& Bouchet 1990). Detections were also reported at $350 \mathrm{~d}$ (Meikle et al. 1993) and $\sim 500$ days after the outburst (see e.g. Bouchet \& Danziger 1993). Signatures of dust were evident in the type IIP SN 1999em after $\sim 450 \mathrm{~d}$ (Elmhamdi et al. 2003) and also in SN 2004et starting at 300 days (Sahu et al. 2006; Kotak et al. 2009). Additionally, dust formation in the type Ib SN 1990I was reported to have commenced as early as $\sim 250 \mathrm{~d}$ 
(Elmhamdi et al. 2004). However, at the latest epochs under consideration here, we can conclusively state that signatures of dust formation in the ejecta of SN 2007gr are not apparent. Yet, given the rapid evolution of the $\mathrm{SN}$, one might have expected signatures due to dust at epochs earlier than those for SN 1987A, and other type II-P SNe.

\subsection{Estimate of the Fe mass}

The very late-time near-IR spectrum at $+376.7 \mathrm{~d}$ (Fig. 12) represents the only one of its kind for type Ic SNe.

As SN 2007 gr evolves into its super-nebular phase, the rapid decrease in the electron temperature and density of the ejecta implies that strong [Fe II] and [Si I] lines in the $J$ and $H$ bands should be present. This is borne out by the observations with the prominent feature at $\sim 1.65 \mu \mathrm{m}$ either arising from [Fe II] $1.6435 \mu \mathrm{m}\left(\mathrm{a}^{4} \mathrm{~F}-\mathrm{a}^{4} \mathrm{D}\right)$ or [Si I] $1.6454 \mu \mathrm{m}$, or even a blend of both. To further add to the complexity of this scenario, the intensity ratio between the lines of these ions (i.e. [Fe II] 1.6454, $1.6068 \mu \mathrm{m}$ and [Si I] $1.6435,1.5994 \mu \mathrm{m})$ are expected to be similar at high densities (see Oliva et al. 1987).

However, if this feature is mainly due to [Fe II] then we should be able to detect also the $1.257 \mu \mathrm{m}\left(\mathrm{a}^{6} \mathrm{D}-\mathrm{a}^{4} \mathrm{D}\right)$ transition, which shares the same upper level with the $1.6435 \mu \mathrm{m}$ emission line. In our late-time spectrum, we do not clearly detect the $1.257 \mu \mathrm{m}$ line which should, in fact, have an intensity $\sim 1.35$ times greater than the $1.6435 \mu \mathrm{m}$ line (Meikle et al. 1989). Alternatively, if we assume [Si I] dominates the contribution to the $1.65 \mu \mathrm{m}$ feature, we expect to observe the strongest near-IR transition of $[\mathrm{SiI}]$ at $1.0991 \mu \mathrm{m}$. Thus the feature at $\sim 1.1 \mu \mathrm{m}$ may be mainly attributed to [Si I]. The identification of $[\mathrm{SiI}]$ is supported by the absence of a weaker [Fe II] emission at $1.68 \mu \mathrm{m}$ ( $\sim 25 \%$ the flux of [Fe II] $1.6454 \mu \mathrm{m})$.

For the purpose of estimating an upper limit to the mass of ${ }^{56} \mathrm{Fe}$ ejected in SN 2007gr, we have used the earlier spectrum at $+175 \mathrm{~d}$ where the identification of the $1.65 \mu \mathrm{m}$ feature with $[\mathrm{Fe} \mathrm{II}]$ is more secure and the $\mathrm{S} / \mathrm{N}$ is much higher.

The quantity of iron in the ejecta can be derived if we know the temperature, density and emissivity of the [Fe II] emission regions (Graham et al. 1986). Therefore models which consider the ionisation structure of the SN ejecta are necessary to determine an accurate iron mass at this epoch. Graham et al. (1986) related the 1.6435 micron line flux to the iron mass $\left(M_{\mathrm{Fe}}\right)$ for the type Ib SN $1983 \mathrm{~N}$ according to the equation:

$$
F_{1.644 \mu \mathrm{m}}=\frac{A_{\mathrm{a}_{4} \mathrm{~F}_{9 / 2}-\mathrm{a}_{4} \mathrm{D}_{7 / 2}} h v}{4 \pi d^{2}} \frac{n_{\mathrm{a}^{4} \mathrm{D}_{7 / 2}}}{n_{\mathrm{Fe} \mathrm{II}}} \frac{n_{\mathrm{Fe} I \mathrm{I}}}{n_{\mathrm{Fe}}} \frac{M_{\mathrm{Fe}}}{m_{\mathrm{Fe}}} \exp \left(-\tau_{\mathrm{H}}\right)
$$

where $A$ denotes the Einstein spontaneous decay probability $\left(0.00569 \mathrm{~s}^{-1}\right.$; Nussbaumer \& Storey 1982), $h v$ is the energy of the transition (i.e. $\mathrm{a}_{4} \mathrm{~F}_{9 / 2}-\mathrm{a}_{4} \mathrm{D}_{7 / 2}$ ), and $d$ is the distance to the $\mathrm{SN}$. The optical depth of the line is represented by $\tau_{\mathrm{H}}$, and $m_{\mathrm{Fe}}$ denotes the mass of an iron atom. Graham et al. (1986) estimated that the ejecta of SN $1983 \mathrm{~N}$ contained $\sim 0.3 M_{\odot}$ of iron.

To obtain an estimate for SN 2007gr, we have adopted similar physical parameters. Assuming LTE conditions, the forbidden lines are excited at temperatures $\sim 6500 \mathrm{~K}$, and so $n_{a^{4} \mathrm{D}_{7 / 2}} / n_{\mathrm{Fe}} \approx 0.03$. This assumption is valid for our $+176 \mathrm{~d}$ spectrum since electron densities are typically $>10^{6} \mathrm{~cm}^{-3}$ before 200 days (Graham et al. 1986).

Since our spectrum was observed $\sim$ half a year from $B$ maximum, the ionisation fraction $n_{\mathrm{Fe} \mathrm{II}} / n_{\mathrm{Fe}}$ can be approximated as 0.1 (Meyerott 1980; Axelrod 1980). Taking into account the distance to SN 2007gr and a negligible extinction for the
$1.6435 \mu \mathrm{m}$ line in the $H$ band (see Table 1), the integrated line flux of $\sim 6 \times 10^{-15} \mathrm{erg} \mathrm{cm}^{-2} \mathrm{~s}^{-1}$ implies that $0.14 \pm 0.08 M_{\odot}$ of Fe was synthesised in SN 2007 gr. Since the quantity of ${ }^{56} \mathrm{Ni}$ derived from the bolometric light curve is $0.076 \pm 0.010 M_{\odot}$, the iron mass is a plausible upper limit. The error in the estimate of Fe accounts for the uncertainties in the distance to SN 2007gr, the extinction and also on the atomic data.

\section{Summary}

We have presented an extensive set of optical and near-IR observations of the type Ic SN 2007gr. In order to put SN 2007gr into context, we compared our data with a sample of relatively wellmonitored type Ic SNe that span a range in observed properties. The light curves of SN 2007gr best resemble those of the broadlined ("hypernova") type Ic SN 2002ap at early times, peaking with a $B$ band magnitude of $M_{B}=-16.8$. However, the peak absolute optical magnitudes lie towards the fainter end of the Ic $\mathrm{SNe}$ that we considered here.

The narrow spectral features of SN 2007gr compared to typical $\mathrm{SNe} \mathrm{Ib} / \mathrm{c}$, are indicative of low expansion velocities, and facilitate line-identification which is usually problematic in type Ic SNe due to severe line-blending. The similarity with SN 2002ap does not extend to the spectroscopic observations, with SN 1994I generally providing a better match.

Our nebular phase spectra reveal a systematic blue-shift in the profiles of forbidden [O I] and $\mathrm{MgI}$, while those of the permitted lines of the same elements are centred at their rest wavelengths. This implies that the forbidden and permitted lines appear in different regions of the ejecta, as expected. The systematic blue-shift of the forbidden lines is most likely due to the absorption of photons emitted on the far side of the SN by the intervening, higher opacity material.

We found low $\mathrm{MgI}] /[\mathrm{OI}]$ ratios for SN 2007gr during the early nebular phase supporting the previous suggestion that the progenitor was a carbon-rich star (Valenti et al. 2008a). This finding, taken together with the comparisons with SN 2002ap, and the relatively rapid spectral evolution e.g. the early appearance of $\mathrm{CO}$, suggest a highly-evolved, compact progenitor. From modelling of the quasi-bolometric light curve, we estimate that a modest $2-3.5 M_{\odot}$ of material was ejected by SN $2007 \mathrm{gr}$ at a kinetic energy of $1-4$ foe, and $0.076 \pm 0.020 M_{\odot}$ of ${ }^{56} \mathrm{Ni}$ was synthesised in the explosion - with the latter value being very similar to that found for SN 2002ap.

We clearly detected the first-overtone of the $\mathrm{CO}$ molecule and were able to track its evolution. It first appeared about day +71 - the earliest recorded appearance of this molecule and persisted for at least $100 \mathrm{~d}$.

The proximity of SN $2007 \mathrm{gr}$, one of the nearest SNe Ic in recent years, has provided us with an ideal target for intensive follow-up. Indeed, SN 2007gr is the first type Ic SN for which near-IR spectra have been obtained at all phases. We suggest that SN 2007gr is an ideal template for future $\mathrm{SNe}$ Ib/c studies.

Acknowledgements. We would like to thank all the staff from the Asiago Ekar Telescope, Campo Imperatore Telescope, Calar Alto Observatory, Gemini North Telescope, Nordic Optical Telescope (operated on the island of La Palma jointly by Denmark, Finland, Iceland, Norway, and Sweden), Osservatorio di Teramo, Sternberg Astronomical Institute Telescope, Telescopio Nazionale Galileo, UKIRT, and the Wendelstein Telescope. The Gemini data reported here were obtained via programmes GN-2007B-DD-3 and GN-2008B-Q-58.

We are grateful to the staff at the NOT: Amanda Djupvik, Thierry Morel, Jarkko Niemela, Tapio Pursimo, Auni Somero, John Telting and Helena Uthas, for observing SN 2007gr. We would also like to thank the staff at Gemini North: Thomas Dall, Tom Geballe, Silas Laycock, Atsuko Nitta, Kathy Roth, Ricardo Schiavon, Chad Trujillo and Kevin Volk, and also to the observers at 
the TNG: Avet Harutyunyan, Lorenzi Vania, and the Wendelstein observatory: Remus Bergemann, Florian Lang and Johannes Koppenhoefer.

J. Fynbo acknowledges the Dark Cosmology Centre which is supported by the DNRF; R.K. and S.J.S acknowledge financial support from STFC. V. Stanishev acknowledges financial support from the Fundação para a Ciência e a Tecnologia.

This paper has made use of data obtained from the Isaac Newton Group Archive which is maintained as part of the CASU Astronomical Data Centre at the Institute of Astronomy, Cambridge. This research has made use of the NASA/IPAC Extragalactic Database (NED) which is operated by the Jet Propulsion Laboratory, California Institute of Technology, under contract with the National Aeronautics and Space Administration. This publication has made use of data products from the Two Micron All Sky Survey, which is a joint project of the University of Massachusetts and the Infrared Processing and Analysis Center/California Institute of Technology, funded by the National Aeronautics and Space Administration and the National Science Foundation.

\section{References}

Alard, C. 2000, A\&AS, 144, 363

Alard, C., \& Lupton, R. 1998, ApJ, 503, 325

Arnett, D., Supernovae and Nucleosynthesis 1996 (Princeton, NJ: Princeton University Press), 414

Arnett, W. D. 1982, ApJ, 253, 785

Axelrod, T. S. 1980, Ph.D. Thesis, UCRL52994, University of California

Baron, E., Hauschildt, P. H., Branch, D., Kirshner, R. P., \& Filippenko, A. V. 1996, MNRAS, 279, 779

Benetti, S., Cappellaro, E., Turatto, M., et al. 2006, ApJ, 653, L129

Bouchet, P., \& Danziger, I. J. 1993, A\&A, 273, 451

Burstein, D., \& Heiles, C. 1984, ApJS, 54, 33

Cappellaro, E., Mazzali, P. A., Benetti, S., et al. 1997, A\&A, 328, 203

Cardelli, J. A., Clayton, G. C., \& Mathis, J. S. 1989, ApJ, 345, 245

Catchpole, R., \& Glass, I. 1987, IAU Circ., 4457

Chornock, R., Filippenko, A. V., Li, W., et al. 2007, CBET, 1036, 1

Clayton, D. D., Liu, W., \& Dalgarno, A. 1999, Science, 283, 1290

Clocchiatti, A., \& Wheeler, J. C. 1997, ApJ, 491, 375

Clocchiatti, A., Wheeler, J. C., Brotherton, M. S., et al. 1996, 462

Crockett, R. M., Maund, J. R., Smartt, S. J., et al. 2008, ApJ, 672, L99

Dalgarno, A., Stancil, P. C., \& Lepp, S. 2008, Stellar Evolution, Stellar Explosions and Galactic Chemical Evolution, Proceedings of the 2nd Oak Ridge Symposium on Atomic and Nuclear Astrophysics

Elmhamdi, A., Danziger, I. J., Chugai, N., et al. 2003, MNRAS, 338, 939 Elmhamdi, A., Danziger, I. J., Cappellaro, E., et al. 2004, A\&A, 426, 963 Fassia, A., Meikle, W. P. S., Chugai, N., et al. 2001, MNRAS, 325, 907 Filippenko, A. V., \& Sargent, W. L. 1986, Nature, 316, 407

Filippenko, A. V., Barth, A. J., Bower, G. C., et al. 1995a, AJ, 110, 2261 Filippenko, A. V., Barth, A. J. Matheson, T., et al. 1995b, ApJ, 450, L11 Folatelli, G., Contreras, C., Phillips, M., et al. 2006, ApJ, 641, 1039

Foley, R. J., Papenkova, M. S., Swift, B. J., et al. 2003, PASP, 115, 1220 Fransson, C., Challis, P. M., Chevalier, R. A., et al. 2005, ApJ, 622, 991 Galama, T. J., Vreeswijk, P. M., van Paradijs, J., et al. 1998, Nature, 395, 670 Gal-Yam, A., Ofek, E., \& Shemmer, O. 2002, MNRAS, 332L, 73G Gearhart, R. A., Wheeler, J. C., \& Douglas, A. S. 1999, ApJ, 510, 944 Gerardy, C. L., Fesen, R. A., Höflich, P., et al. 2000, AJ, 119, 2968

Gerardy, C. L., Fesen, R. A., Nomoto, K., et al. 2002, Publ. Astron. Soc. Jpn, 54, 905

Goodrich, R. W., Stringfello, G. S., \& Penrod, G. D. 1989, ApJ, 342, 908

Graham, J. R., Meikle, W. P. S., Allen, D. A., Longmore, A. J., \& Williams, P. M. 1986, MNRAS, 218, 93

Gyoon, L. M., Eunhyeuk, K., Sang Chul, K., et al. 1995, J. Kor. Astron. Soc., 28,31

Hamuy, M., Maza, J., Pinto, P. A., et al. 2002, ApJ, 124, 417

Hachisu, I., Matsuda, T., Nomoto, K., \& Shigeyama, T. 1991, ApJ, 368, L27

Hjorth, J., Sollerman, J., Moller, P., et al. 2003, Nature, 423, 6942, 847

Iwamoto, K., Nomoto, K., Hölich, P., et al. 1994, ApJ, 437, L115

Iwamoto, K., Mazzali, P. A., Nomoto, K., et al. 1998, Nature, 395, 672

Kinugasa, K., Kawakita, H., Ayani, K., et al. 2002, ApJ, 577, L97

Kotak, R., Meikle, W. P. S., Van Dyk, S. D., et al. 2005, ApJ, 628, L123

Kotak, R., Meikle, W. P. S., Pozzo., M., et al. 2006, ApJ, 651, L117

Kotak, R., Meikle, W. P. S., Farrah, D., et al. 2009, ApJ, 704, 306

Kozma, C., \& Fransson, C. 1998, ApJ, 496, 946

Lee, M. G., Kim, E., Kim, S. C., et al. 1995, J. Kor. Astron. Soc., 28, 31

Lepp, S., Dalgarno, A., \& McCray, R. 1990, ApJ, 358, 262

Liu, W., \& Dalgarno, A. 1995, ApJ, 454, 472

Liu, W., Dalgarno, A., \& Lepp, S. 1992, ApJ, 385, 240

Liu, W., Jeffrey, D. J., \& Schultz, D. R. 1998, ApJ, 494, 812

Madison, D., \& Li, W. 2007, CBET, 1034, 1
Maeda, K., Mazzali, P. A., \& Nomoto, K. 2006, ApJ, 645, 1331

Maeda, K., Kawabata, K., Mazzali, P. A., et al. 2008, Science Express, 319, 1220

Malesani, D., Fynbo, J. P. U., Hjorth, J., et al. 2009, ApJ, 692, 2, L84

Mazzali, P. A., \& Lucy, L. B. 1998, MNRAS, 295, 428.

Mazzali, P. A., Iwamoto, K., \& Nomoto, K. 2000, ApJ, 545, 407M.

Mazzali, P. A., Nomoto, K., Patat, F., \& Maeda, K. 2001, ApJ, 559, 1047

Mazzali, P. A., Deng, J., Maeda, K., et al. 2002, ApJ, 572, L6

Mazzali, P. A., Deng, J., Tominaga., N., et al. 2003, ApJ, 599, L95

Mazzali, P. A., Kawabata, K. S., Maeda, K., et al. 2005, Science, 308, 1284

Mazzali, P. A., Foley, R., \& Deng, J., et al. 2007, ApJ, 661, 892

Mazzali, P. A., Valenti, S., \& Della Valle, M., et al. 2008, Science, 321, 1185

McKenzie, E. H., \& Schaefer, B. 1999, PASP, 111, 964

Meikle, W. P. S. 1989, MNRAS, 238, 193

Meikle, W. P. S., Spyromilio, J., Allen, D. A., et al. 1993, MNRAS, 261, 535

Meikle, W. P. S., Cumming, R. J., Geballe, T. R., et al. 1996, 281, 263

Meyerott, R. E. 1980, ApJ, 239, 257

Milisavljevic, D., Fesen, R. A., Gerardy, C. L., et al. 2009

[arXiv:0904.4256v1]

Milliard, J., Branch, D., Baron, E., et al. 1999, ApJ, 527, 746

Modjaz, M., Kirshner, R. P., Challis, P., \& Matheson, T., 2008, ApJ, 687, L9

Nakamura, T., Mazzali, P. A., Nomoto, K., \& Iwamoto, K. 2001, ApJ, 550, $991 \mathrm{~N}$

Nomoto, K., Filippenko, A. V., \& Shigeyama, T. 1990, A\&A, 240, L1

Nomoto, K., Yamaoka, H., Pols., O. R., et al. 1994, Nature 371, 227

Oliva, E., Moorwood, A. F. M., \& Danziger, I. J. 1987, ESO Messenger, 50, 18

Pandey, S. B., Anupama, G. C., Sagar, R., et al. 2003, MNRAS, 340, 375

Patat, F. 1996, Ph.D. Thesis, Univ. Padova

Patat, F., Cappellaro, E., Danziger, J., et al. 2001, ApJ, 555, 900P

Petuchowski, S. J., Dwek, E., Allen, J. E., \& Nuth, J. A. 1989, ApJ, 342, 406

Pignata, G., Patat, F., Benetti, S., et al. 2004, MNRAS, 335, 178

Pignata, G., Benetti, S., Mazzali, P. A., et al. 2008, MNRAS, 388, 971

Podsiadlowski, P., Joss, P. C., \& Hsu J. J. L. 1992, ApJ, 391, 246

Pols, O. R., \& Nomoto, K. 1997, ASP Conf. Ser., 130

Richmond, M. W., van Dyk, S. D., Ho, W., et al. 1996, AJ, 111, 327

Sahu, D. K., Anupama, G. C., Srividya, S., \& Muneer, S. 2006, MNRAS, 372, 1315

Sauer, D. N., Mazzali, P. A., Deng, J., et al. 2006, MNRAS, 369, 1939.

Schlegel, D. J., Finkbeiner, D. P., \& Davis, M. 1998, ApJ, 500, 525

Sharp, C., \& HŽflich, P. 1989, Highlights of Astronomy, ed. D. McNally, IAU

General Assembly (Dordrecht: Kluwer), 8, 207

Shigeyama, T., Nomoto, K., Tsujimoto, T., \& Hash, M. 1990, ApJ, 361, L23

Silbermann, N. A., Harding, P., Madore, B. F., et al. 1996, ApJ, 470, 1

Silverman, J. M., Mazzali, P., Chornock, R., et al. 2009, PASP, 121, 689

Soderberg, A. M., Berger, E., Page, K. L., et al. 2008, Nature, 453, 7194, 469

Sollerman, J., Kozma, C., Fransson, C., et al. 2000, ApJ, 537, L127

Spyromilio, J. 1991, MNRAS, 253, L25

Spyromilio, J., \& Leibundgut, B. 1996, MNRAS, 283, L89

Spyromilio, J., Meikle, W. P. S., Allen, D. A., \& Learner, R. C. M. 1988, Nature, 334,327

Spyromilio, J., Leibundgut, B., \& Gilmozzi, R. 2001, A\&A, 376, 188

Stritzinger, M., Humay, H., Suntzeff, N. B., et al. 2002, ApJ, 124, 2100

Stritzinger, M., Mazzali, P., Phillips, M., et al. 2009, ApJ, 696, 713

Suntzeff, N. B., \& Bouchet, P. 1990, AJ, 99, 650

Sutherland, P. G., \& Wheeler, J. C. 1984, ApJ, 280, 282

Swartz, D. A., Filippenko, A. V., Nomoto, K., \& Wheeler, J. C. 1993, ApJ, 411, 313

Tanaka, M., Kawabata, K. S., Maeda, K., Hattori, T., \& Nomoto, K. 2008, ApJ, 689,1191

Taubenberger, S., Pastorello, A., \& Mazzali, P. A., et al. 2006, MNRAS, 371, 1459-1477.

Taubenberger, S., Valenti, S., Benetti, E., et al. 2009, MNRAS, 397, 2, 677

Terry, J. N., Paturel, G., \& Ekholm, T. 2002, A\&A, 393, 57

Tomita, H., Deng, J., Maeda, K., et al. 2006, ApJ, 644, 400

Tsvetkov, D. Yu., \& Pavlyuk, N. N. 1995, Astron. Lett., 21, 606

Valenti, S., Elias-Rosa, N., Taubenberger, S., et al. 2008a, ApJ, 673, L155

Valenti, S., Benetti, S., Capellaro, E., et al. 2008b, MNRAS, 383, 1485

Valenti, S., Pastorello, A., Cappellaro, E., et al. 2009, Nature, 459, 674

Wheeler, J. C., \& Levreault, R. 1985, ApJ, 294, L17

Wheeler, J. C., Harkness, R. P., Barker, E. S., Cochran, A. L., \& Wills, D. 1987, ApJ, 313, L69

Wheeler, J. C., Swartz, D. A., \& Harkness, R. P. 1993, Phys. Rep., 227, 113

Wheeler, J. C., Hoeflich, P., Harkness, R. P., \& Spyromilio, J. 1998, ApJ, 496, 908

Wooden, D. H., Rank, D. M., Bregman, J. D., \& Witteborn, F. C. 1993, ApJS, 88,477

Woosley, S. E., \& Bloom, J. S. 2006, ARA\&A, 44, 507

Yokoo, T., Arimoto, J., Matsumoto, K., \& Takahashi, A. 1994, PASJ, 46, L191

Yoshii, Y., Tomita, H., Kobayashi, Y., et al. 2003, ApJ, 592, 467 